\title{
L'artisanat tuilier-briquetier dans le canton des Herbiers (Vendée) à l'époque contemporaine : méthodologie et perspectives de recherche
}

The tile-brick making in the canton of Les Herbiers (Vendée) during the contemporary period: methodology and research perspectives

La producción artesanal de tejas y ladrillos en el cantón de Les Herbiers (Vendée) en época contemporánea: metodología y perspectivas de investigación Das Ziegeleihandwerk im Bereich des Kantons „Les Herbiers“ in der Vendée: methodisches Vorgehen und Forschungsperspektiven

\section{Guillaume Chamarre}

\section{OpenEdition}

Journals

Édition électronique

URL : https://journals.openedition.org/rao/7439

DOI : $10.4000 /$ rao. 7439

ISSN : $1775-3732$

Éditeur

Presses universitaires de Rennes

Édition imprimée

Date de publication : 21 décembre 2021

Pagination : 301-321

ISBN : 978-2-7535-8668-0

ISSN : 0767-709X

\section{Référence électronique}

Guillaume Chamarre, «L'artisanat tuilier-briquetier dans le canton des Herbiers (Vendée) à l'époque contemporaine : méthodologie et perspectives de recherche », Revue archéologique de l'Ouest [En ligne], 37 | 2021, mis en ligne le 21 décembre 2021, consulté le 10 octobre 2022. URL : http:// journals.openedition.org/rao/7439; DOI : https://doi.org/10.4000/rao.7439 


\title{
L'artisanat tuilier-briquetier dans le canton des Herbiers (Vendée) à l'époque contemporaine : méthodologie et perspectives de recherche
}

\author{
The tile-brick making in the canton of Les Herbiers (Vendée) \\ during the contemporary period: methodology and research perspectives
}

\author{
Guillaume Chamarre \\ (chamarre.guillaume@orange.fr)
}

\begin{abstract}
Résumé : Cet article présente les principaux résultats d'une étude menée à l'occasion de deux années de master à l'université de Nantes, sous la direction de Yves Henigfeld, Jean-François Nauleau et Alain Champagne. Une approche diachronique a permis d'appréhender l'artisanat tuilier-briquetier, du XvII ${ }^{e}$ s. au Xxe s., sur le canton des Herbiers (Vendée) [Chamarre, 2018; Chamarre, 2019]. Un dépouillement exhaustif des supports cartographiques et des sources archivistiques, successivement fournis par les fonds seigneuriaux et ecclésiastiques sous l'Ancien Régime puis par l'administration publique dès le $\mathrm{XIX}^{\mathrm{e}} \mathrm{s}$., a ainsi permis l'observation d'une tradition artisanale croissante jusqu'au milieu du $\mathrm{XIX}^{\mathrm{e}}$ s., avant un déclin constant jusqu'au milieu du $\mathrm{XX}^{\mathrm{e}} \mathrm{s}$. Cette dynamique continue à l'époque contemporaine est perçue non seulement au travers du recensement et du suivi d'une multitude de petits ateliers familiaux, implantés dans une sphère économique locale, mais également au travers d'une contrainte économique croissante ayant influé sur les choix de production spécialisée ou encore les politiques d'embauche des femmes et des enfants. L'étude a également mesuré l'intérêt méthodologique des approches par le biais des matériaux de construction contemporains et du témoignage des anciens exploitants sur site. Les principaux résultats dressent un aperçu des acquis méthodologiques les plus appropriés à l'étude des tuileries-briqueteries contemporaines.
\end{abstract}

\begin{abstract}
This paper presents an overview of main results of a study carried out during a Master's degree at the University of Nantes, under the direction of Yves Henigfeld, Jean-François Nauleau and Alain Champagne. This diachronic study focused on tile-brick making from the $17^{\mathrm{th}}$ to $20^{\text {th }}$ century, in the Canton of Les Herbiers (Vendée, France) (Chamarre, 2018; Chamarre, 2019). This work, which involved the study of maps and archives from local ecclesial communities, seigneurial system under the Ancien Régime and public records from the 19th century onwards, has revealed a sustained expansion of this craft before the middle of 19 th century, then a decline until the middle of $20^{\text {th }}$ century. This evolution is documented from a census of the many family workshops of the area and by studying the strategies that allowed the tile-brick craft to adapt as well as the hiring of women and children. The survey also measured the pertinence of the study of contemporary building materials and the testimony of former operators on site. The main results give an overview of the methodological achievements in the study of contemporary brickworks.
\end{abstract}

Mots clés: Vendée, époque contemporaine, atelier, exploitation, tuile, recensement, archives, méthodologie, tuilerie, briqueterie.

Keywords: Vendée, contemporary period, workshop, operation, tile, census, archives, methodology, tile factory, brick factory.

La vulnérabilité des vestiges de l'artisanat tuilier-briquetier de l'époque contemporaine détonne avec la multiplication des études traitant des matériaux de construction en terre cuite appliqués au contexte archéologique pour les périodes antiques et médiévales. Ce constat encourage à la systématisation du recensement des tuileries-briqueteries et de leur étude archivistique et archéologique.
La reconnaissance d'une tradition artisanale tuilière dès l'époque moderne, et à l'œuvre jusqu'au XIX ${ }^{\mathrm{e}}$ et $\mathrm{xx}^{\mathrm{e}}$ s., à l'échelle de l'arrondissement de La Roche-surYon, et plus particulièrement sur le district cantonal des Herbiers (Vendée) ${ }^{1}$ (fig. 1), a justifié l'application d'un

1. Le territoire retenu pour cette étude correspond à la circonscription de 18242015, constitué initialement de 10 communes : Ardelay, Beaurepaire, Les Epesses, 


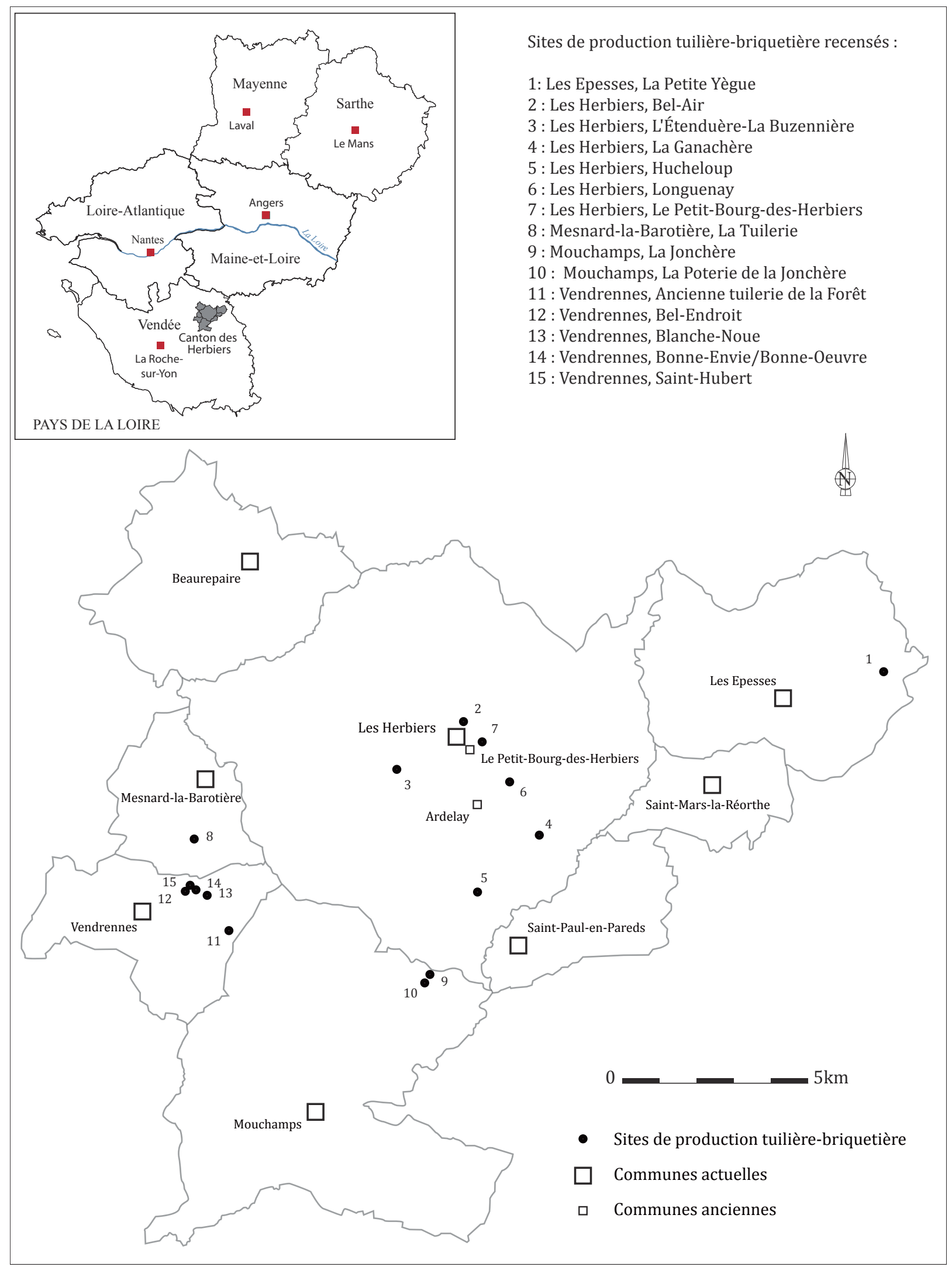

Figure 1: Carte de répartition des tuileries-briqueteries recensées, du XVII au XXe S., dans le canton des Herbiers (Vendée).

Figure 1: Distribution map of tile-brick factories, from 17th to 20th century, in the canton of Les Herbiers (Vendée). 
dépouillement exhaustif des supports cartographiques et des sources archivistiques produits sous l'Ancien Régime puis l'administration préfectorale. Parmi les principaux champs d'études abordés, la quantification et le recensement des ateliers ont été associés à un suivi de la maind'œuvre tuilière et des productions quantitativement fluctuantes, de l'extrême fin du $\mathrm{XvIII}^{\mathrm{e}} \mathrm{s}$. au milieu du $\mathrm{Xx}^{\mathrm{e}} \mathrm{s}$.

Un certain nombre de données ont également été acquises par l'enregistrement de matériaux de construction contemporains.

La confrontation de ces diverses approches méthodologiques ainsi que des problématiques macro- et microéconomiques associées aux ateliers et à leur main-d'œuvre constitue le point essentiel de cette étude.

\section{LES ANTÉCÉDENTS MODERNES D'UN ARTISANAT TUILIER-BRIQUETIER (XVII S.-FIN XVIII' S.)}

L'artisanat tuilier-briquetier contemporain hérite d'une tradition artisanale attestée sous l'Ancien Régime. Un dépouillement des archives anciennes s'est principalement intéressé aux fonds seigneuriaux et ecclésiastiques. Prenant essentiellement la forme d'aveux et titres de propriété, ces archives anciennes font mention d'ateliers appartenant à des seigneurs laïcs et à des communautés ecclésiastiques (tabl. 1).

Ces « ateliers seigneuriaux » ne sont pas exploités par les propriétaires mais sont confiés à des artisans sur un schéma de faire-valoir indirect. L'artisan peut être lié à l'atelier seigneurial par un contrat d'embauche, un bail à ferme (affermage) ou un droit de jouissance de longue durée voire perpétuel (accensement). Au même titre que les exploitations agricoles d'un domaine foncier seigneurial, dont une part des profits tirés des activités lucratives réalisées revient au seigneur, une redevance sur la location de l'atelier fieffé peut être perçue en argent et/ ou en nature. Par la signature du bail, renouvelable ou non reconductible à l'échéance, le bailleur peut ainsi s'octroyer un moyen d'approvisionnement en matériaux (Champagne, 2007).

Néanmoins, toute une frange artisanale ne transparaît pas dans ces archives anciennes : les ateliers indépendants. Ces derniers sont la propriété d'un artisan ou d'un collectif d'artisans, propriétaires de leurs propres outils de travail. Cette frange de l'artisanat tuilier échappe aux inventaires et fonds seigneuriaux. La part de ces ateliers indépendants ne doit pas être sous-estimée, comme cela a déjà pu être souligné pour le Haut-Poitou (Champagne, 2007).

Les Herbiers, Mesnard-la-Barotière, Mouchamps, Le Petit-Bourg-des-Herbiers, SaintMars-la-Réorthe, Saint-Paul-en-Pareds et Vendrennes.
Malgré le suivi de quelques ateliers et de leurs exploitants dès 1644 , le seul dépouillement de ces baux et titres ne permet pas la localisation exacte des ateliers mentionnés.

\section{Antécédents d'une activité tuilière à La Jonchère}

Plusieurs mentions d'ateliers de tuiliers apparaissent dans les fonds seigneuriaux et ecclésiastiques dépouillés, témoignant toutes d'un artisanat tuilier développé au lieudit de La Jonchère.

Ces déclarations correspondent pour une part à des titres détenus par l'abbaye Notre-Dame de la Grenetière (Grainetière). L'abbaye est située sur la paroisse d'Ardelay, en lisière septentrionale de la forêt du parc Soubise, à près de 3,5 km du village de La Jonchère.

Un bail signé à la date du 7 décembre 1644 fait ainsi mention d'une " portion de la terre des Pleisses, dans laquelle portion sont les terres où les dits déclarants [Jacques et Charles Guymard, demeurant à La Jonchère] prennent terres pour faire thuilles ». Une annotation précise l'existence d'une " boisselée et demi de terre propres à faire thuilles sises aux Grandes Pleisses [à la Pitancerie] ${ }^{2}$ ». Ce bail s'insère parmi les divers titres de l'abbaye.

À la date du 17 janvier 1671, une pièce de terre inculte est cédée à un dénommé Michel Goislard, marchand demeurant au lieu-dit de La Jonchère. Les termes du bail stipulent un droit de faire bâtir une maison et grange, à la seule condition "de ne point souffrir que personne puisse tirer terre en la dite pièce pour faire thuilles si ce n'est les dit Abbé et religieux à qui il sera permis et le faire jusqu'à ce que le dit terrain soit propre à autre usage ". Le droit de tirage d'une terre à faire tuiles sur le terrain concerné était alors réservé à $\mathrm{M}^{\mathrm{gr}} \mathrm{J}$ Jean Birocheau, abbé à la Grainetière, et aux membres de l'abbaye, jusqu'à ce que la parcelle soit aménagée ${ }^{3}$.

La relation étroite entre une exploitation tuilière-briquetière au lieu-dit de La Jonchère et l'abbaye est réaffirmée dans un bail à rente daté du 9 juin 1671. Ce bail mentionne un "petit Bordage appelé La Batarderie situé au village de la Jonchère paroisse de Mouchamps consistant en une petite maison appentis, grange, et fourneau à thuilles chaux et briques, et deux jardins avec les appartenances ». Le bail passe à Jean Chateigner, Jacques Chateigner, Louise Chateigner et Jean Mignonneau, " moyennant soixante sols de rente féodale et foncière, payable le jour de noël en la dite Abbaye et [...] de payer au sacristain et ses successeurs religieux en la dite Abbaye à perpétuité deux livres de cire au premier février, et encore aux conditions

2. Archives départementales d'Eure-et-Loir, $\mathrm{H} 4031$ : Inventaire des titres et papiers de l'abbaye de la Grainetière (XII'-xVIII S.) (1783), [en ligne], p. 117-273.

3. Archives départementales d'Eure-et-Loir, $\mathrm{H}$ 4031: Inventaire des titres et papiers de l'abbaye de la Grainetière (XII'-XVIII S.) (1783), [en ligne], p. 115-116 à 273. 


\begin{tabular}{|c|c|c|c|c|}
\hline Fonds & Période & \multicolumn{2}{|r|}{ Description } & Cote en archives \\
\hline \multirow{2}{*}{ Chartrier d'Ardelay } & \multirow{2}{*}{$1659-1698$} & Tome II & \multirow{2}{*}{$\begin{array}{l}\text { Baux et déclarations à la } \\
\text { baronnie d'Ardelay }\end{array}$} & $\begin{array}{l}\text { Arch. dép. Vendée, } 42 \mathrm{~J} 12 \text {, } \\
\text { [consultation aux archives] }\end{array}$ \\
\hline & & Tome III & & $\begin{array}{l}\text { Arch. dép. Vendée, } 42 \mathrm{~J} 13 \text {, } \\
\text { [consultation aux archives] }\end{array}$ \\
\hline \multirow{3}{*}{ Titres de l'Étenduère } & $1375-1630$ & Tome I & \multirow{3}{*}{$\begin{array}{l}\text { Inventaire et suivi de } \\
\text { l'exploitation des pièces }\end{array}$} & Arch. dép. Vendée, 32 J 12 \\
\hline & $1631-1784$ & Tome II & & Arch. dép. Vendée, 32 J 13, [en ligne] \\
\hline & $1589-1789$ & Tome III & & Arch. dép. Vendée, 32 J 14, [en ligne] \\
\hline $\begin{array}{c}\text { Titres et papiers de l'abbaye } \\
\text { de la Grainetière }\end{array}$ & $\mathrm{XII}^{\mathrm{e}}-\mathrm{XVIII}{ }^{\mathrm{e}} \mathrm{s}$ & \multicolumn{2}{|c|}{$\begin{array}{c}\text { Copie de } 1001 \text { articles et inventaires de } \\
\text { pièces }(1783)\end{array}$} & $\begin{array}{c}\text { Arch. dép. d'Eure-et-Loir, H 4031, [en } \\
\text { ligne] }\end{array}$ \\
\hline \multirow{13}{*}{$\begin{array}{l}\text { Titres de la baronnie du } \\
\text { Puy-du-Fou }\end{array}$} & $1628-1850$ & \multicolumn{2}{|c|}{$\begin{array}{l}\text { Baux relatifs aux métairies du domaine } \\
\qquad \text { du Puy-du-Fou }\end{array}$} & Arch. dép. Vendée, 33 J 5, [en ligne] \\
\hline & An XI-1842 & \multicolumn{2}{|c|}{$\begin{array}{l}\text { Baux relatifs aux métairies du domaine } \\
\text { du Puy-du-Fou }\end{array}$} & Arch. dép. Vendée, 33 J 6, [en ligne] \\
\hline & $1777-1830$ & \multicolumn{2}{|c|}{$\begin{array}{l}\text { Baux et procès-verbaux de visites de } \\
\text { métairies du domaine du Puy-du-Fou }\end{array}$} & Arch. dép. Vendée, 33 J 7, [en ligne] \\
\hline & $1736-1744$ & \multicolumn{2}{|c|}{ Registre de comptabilité } & Arch. dép. Vendée, 33 J 10, [en ligne] \\
\hline & $1777-1778$ & \multicolumn{2}{|c|}{ Registre de comptabilité } & Arch. dép. Vendée, 33 J 11, [en ligne] \\
\hline & $1786-1787$ & \multicolumn{2}{|c|}{ Registre de comptabilité } & Arch. dép. Vendée, 33 J 12, [en ligne] \\
\hline & $1787-1788$ & \multicolumn{2}{|c|}{ Registre de comptabilité } & Arch. dép. Vendée, 33 J 13, [en ligne] \\
\hline & $1740-1743$ & \multicolumn{2}{|c|}{ Registre de comptabilité } & Arch. dép. Vendée, 33 J 16, [en ligne] \\
\hline & $1744-1745$ & \multicolumn{2}{|c|}{ Registre de comptabilité } & Arch. dép. Vendée, 33 J 17, [en ligne] \\
\hline & $1751-1757$ & \multicolumn{2}{|c|}{ Registre de comptabilité } & Arch. dép. Vendée, 33 J 18, [en ligne] \\
\hline & $1771-1796$ & \multicolumn{2}{|c|}{ Registre de comptabilité } & Arch. dép. Vendée, 33 J 19, [en ligne] \\
\hline & $1796-1842$ & \multicolumn{2}{|c|}{ Registre de comptabilité } & Arch. dép. Vendée, 33 J 20, [en ligne] \\
\hline & $1798-1829$ & \multicolumn{2}{|c|}{ Registre de comptabilité } & Arch. dép. Vendée, 33 J 23, [en ligne] \\
\hline
\end{tabular}

Tableau 1 : Liste des fonds seigneuriaux et ecclésiastiques dépouillés (G. Chamarre).

Table 1: List of seigneurial and ecclesiastical collections that were analysed here (G. Chamarre).

que les dits Abbé religieux et successeurs pourront quand bon leur semblera, faire faire en la dite grange et fours la chaux, thuilles, et briques nécessaires pour l'entretien de leur Abbaye et monastère seulement ». L'abbaye se réserve le droit d'en faire usage et d'en tirer les matériaux nécessaires à son entretien ${ }^{4}$.

À la date du $1^{\text {er }}$ avril 1780, un bail traite d'un pré dit " de Bouchaire ", situé près du village de La Jonchère, devant lequel se positionnaient autrefois " une maison, un four à chaux et thuilles et une halle à faires de la thuilles " ". À la même date du $1^{\text {er }}$ avril 1780 , un énième bail mentionne ce même pré « devant la Bastardière, situé près le village de la Jonchère ou ci devant y avait des maisons, un four à chaux et à thuilles, une aire et une halle, et où de présent n'y a plus qu'une fosse ou réserves pour lequel objet le dit déclarant est redevable envers la dite

4. Archives départementales d'Eure-et-Loir, $\mathrm{H} 4031$ : Inventaire des titres et papiers de l'abbaye de la Grainetière (XII'-XVIII' S.) (1783), [en ligne], p. 107-108 à 273. 5. Archives départementales d'Eure-et-Loir, $\mathrm{H} 4031$ : Inventaire des titres et papiers de l'abbaye de la Grainetière (xII'-xVIII ${ }^{e}$ s.) (1783), [en ligne], p. 221-273. abbaye de trois livres et de deux livres de cire jaune ${ }^{6}$. Les termes du bail semblent correspondre à ceux énoncés dès 1671 pour un petit bordage appelé La Batarderie. Il s'agit vraisemblablement de la même tuilerie, dépendante de l'abbaye de la Grainetière, exploitée en 1671 et dont l'abandon avait rendu les traces visibles ténues dès 1780 .

Le rôle entretenu par la baronnie d'Ardelay dans la pérennité d'une activité tuilière sur ce secteur est également perceptible, comme en atteste une série de baux.

Une déclaration datée du $1^{\text {er }}$ mars 1659 fait mention d'une " maison composée de 2 chambres basses et 2 hautes, moitié d'une grange à thuiles, [...], le tout au village de la Jonchère ». Ces pièces de terre sont déclarées par Jeanne Yvon, veuve d'un dénommé Barthélémy Grillard, auprès de la baronnie d'Ardelay'. Quelques jours plus tard, à la date du 3 mars 1659, une autre déclaration faite

6. Archives départementales d’Eure-et-Loir, $\mathrm{H} 4031$ : Inventaire des titres et papiers de l'abbaye de la Grainetière (XII'-xvIII' s.) (1783), [en ligne], p. 235-273.

7. Archives départementales de Vendée, 44 I 12 : Chartriers d'Ardelay, t II, p. 54-529. 
à la baronnie d'Ardelay par Mathurin Raineau et François Rondeau, mentionne une "partie d'une maison, la moitié d'une grange à faire tuilles appellée la Maison Brûlée, au village de la Jonchère ${ }^{8}$ ». Faute de localisation précise de ces moitiés d'ateliers, ces deux mentions peuvent renvoyer à une seule et même unité de production.

Le maintien d'une activité tuilière sur la commune de Mouchamps, durant le XviII ${ }^{\mathrm{e}}$ s., n'est perçu qu'au travers de quelques mentions.

À la date du 14 avril 1758 est mentionné Charles Briant, tuilier à la tuilerie de l'Estenduère (commune des Herbiers), rendant aveu d'une maison au village de La Jonchère, à laquelle est associée une " aire à faire les tuiles, jouxtant le grand chemin du Boupère à SaintFulgent ». Le nom de ce même propriétaire réapparaît à la date du 14 janvier 1780. Ce tuilier s'avoue sujet immédiat de la châtellenie d'Ardelay et rend compte " de deux chambres de maison, un four, une écurie, une thuilerie et une aire au dit lieu de la Jonchère, joignant d'un côté au midi le dit chemin Saint-Fulgent au Boupère9 ". Cet artisan est alors en possession de deux exploitations tuilières, distantes l'une de l'autre de près de $5 \mathrm{~km}$.

À cette même époque est attribué l'un des premiers éléments de datation d'une tuilerie au lieu-dit de La Poterie de la Jonchère, correspondant à un millésime de 1760, gravé sur une poutre d'un ancien séchoir. Désormais disparu des suites d'un incendie en 1966, ce témoignage constituait l'indice d'une activité tuilière à La Poterie de la Jonchère dès le XviII ${ }^{\mathrm{e}}$ s. (Bossard et al., 2002).

La pérennité d'une tradition tuilière partagée autour des lieux-dits de La Jonchère et de La Poterie de la Jonchère (Mouchamps) tend à s'imposer comme un district artisanal spécialisé jusqu'au $\mathrm{xx}^{\mathrm{e}} \mathrm{s}$., selon le modèle défini par Mathieu Arnoux (Arnoux, 2013). L'implantation d'une tradition tuilière sur ce secteur s'explique par des prédispositions géologiques, avec la proximité d'un interfluve ayant conservé des nappes argileuses à dominantes schisteuses en position primaire affleurante (Goujou, 1994; Gabilly, 1978). Signalé dans le paysage par la forêt de Soubise, ce terroir argileux fut propice à un artisanat céramique, au même titre que les établissements tuiliers de Blanche-Noue, Saint-Hubert et Bonne-CEuvre (commune de Vendrennes), groupés à l'ouest de ce domaine forestier à l'époque contemporaine. Positionné quant à lui à l'est de la forêt de Soubise, le district de La Jonchère s'étend sur deux lieux-dits : La Jonchère et La Poterie de la Jonchère. La poursuite d'une activité tuilière a permis la succession de près de quatre dynasties de tuiliers à La Jonchère (Burcerot/Fonteneau, Chabiron, Rousseau, Véronneau)

8. Archives départementales de Vendée, 44 J 12 : Chartriers d'Ardelay, t II, p. $75-76$ à 529 .

9. Archives départementales de Vendée, $44 \mathrm{I}$ 13: Chartrier d'Ardelay, t III, p. 483-589. jusqu'en 1974, et de deux dynasties de tuiliers à La Poterie de la Jonchère (Burcerot, Rousseau-Charbonneau/Fortin) jusqu'en 1996 (Bossard et al., 2002).

\section{L'ARTISANAT TUILIER-BRIQUETIER CONTEMPORAIN (DÉBUT XIX' S.-MILIEU XXe S.)}

\section{Recensement des ateliers contemporains : approche cartographique}

Le recensement des ateliers au travers des supports cartographiques est régulièrement décrit comme une méthode relativement fiable pour repérer des exploitations tuilières sous l'Ancien Régime (Moulin, 2011; Costa et Robert, 2008) et inversement, peu fiable, concernant des établissements plus tardifs du XIX ${ }^{\mathrm{e}}$ s. (Vue, 2009).

Par souci d'exhaustivité, un recensement cartographique des ateliers tuiliers-briquetiers a été effectué au travers de supports cartographiques s'étendant de la seconde moitié du $\mathrm{XVIII}^{\mathrm{e}} \mathrm{s}$. à la première moitié du $\mathrm{XIX}^{\mathrm{e}} \mathrm{s}$. : cartes de Cassini (dressées entre 1763 et 1786 pour ce territoire $)^{10}$, cadastre parcellaire " primitif » (entre 1808 et 1813), cartes de l'état-major (entre 1841 et 1848$)^{11}$, puis cadastre impérial (du milieu du XIX ${ }^{\mathrm{e}} \mathrm{s}$. ${ }^{12}$. Le recensement toponymique dans ce dernier support a été effectué indépendamment du dépouillement des matrices cadastrales. L'ensemble de l'arrondissement de La Roche-sur-Yon a été abordé par ce biais cartographique.

La terminologie retenue pour ce recensement compte à la fois les termes communément assignés à cette thématique (tuilerie, briqueterie, four à tuiles/briques) ainsi que leurs variantes dialectales. Le terme de telle employé pour désigner la tuile au nord-ouest de la Vendée (Rézeau, 2003) a ainsi été décliné dans le toponyme La Telle, observé à proximité de la tuilerie de La Lévinière, sur la commune des Landes-Génusson ${ }^{13}$. Un autre toponyme, celle de Tartifume, dérivée de l'expression Tard-y-fume, a été employée pour désigner des sites au sein desquels étaient entretenus les foyers de quelques ateliers de tuiliers-briquetiers, parfois sur des temps de cuisson étendus jusqu'en soirée. Ce dialecte attesté en Charente-Maritime, Gironde, Indre-et-Loire, Lot-et-Garonne, Mayenne, Vienne et Maine-et-Loire (Longnon, 1922) a également été recensé sur la commune de Châteauneuf (Vendée) ${ }^{14}$.

10. CASSInI De Thury C.F., Carte générale de la France, secteur Mortagne (17631769 ), feuillet n⿳0 99, secteur Luçon (1763-1769), feuillet n 100 , [en ligne].

11. Carte de l'état-major, 4 EM 130, zone de La Roche-sur-Yon, nord-est (1841), [en ligne].

12. Archives départementales de Vendée, 3P, [en ligne].

13. Cassini de Thury C.F., Carte générale de la France, secteur Mortagne (1763-1769), feuillet $n^{\circ}$ 9g, [en ligne].

14. CASSINI DE ThuRY C.F., Carte générale de la France, secteur Nantes (1783, 1785, 1786), feuillet $n^{\circ} 131$, [en ligne]. 
Quelques termes trahissent également les matériaux du sous-sol favorables à l'installation d'une activité tuilière et briquetière. Parmi ces notions, le terme de noue, employé dès le XIV ${ }^{\mathrm{e}}$ s., dérivé du latin médiéval nauda puis de noe au XIII ${ }^{\mathrm{e}}$ s., désigne entre autres une terre grasse et humide périodiquement inondée ${ }^{15}$. Un autre dérivé linguistique, cette fois du terme argile, a également été assigné à des sites d'extraction argilière. La modification successive $\mathrm{du}$ terme argile en ardille puis ardillier, dont est issu le nom de l'ancienne commune d'Ardelay (Vendée), trouve un cas comparable sous l'appellation des ardriliers signalés aux alentours d'Argentan (Orne) [Moulin, 2011]. Chacun de ces toponymes évocateurs d'une ressource argilière ne sont pas exclusifs à un artisanat tuilier et sont susceptibles d'être associés à un autre artisanat céramique. La proximité de trous d'eau consécutifs du tirage de la terre peut également être retenue pour la reconnaissance d'un artisanat céramique de proximité. De la même manière, les seuls supports cartographiques ne permettent pas une caractérisation des appellations évocatrices de structures de chauffe (four, fournerie, fournil). Cette distinction des unités de cuisson tuilières, de celles potières, chaunières ou domestiques, est permise au travers des états de sections jointes aux matrices du cadastre parcellaire impérial.

À l'issue du recensement des toponymes et des mentions cartographiques relatifs à une activité tuilière, l'approche cartographique apparaît peu fiable notamment à propos de la carte de Cassini et de la carte de l'état-major. De nettes lacunes apparaissent également au travers des relevés cadastraux, "primitif " puis impérial, en comparaison du dénombrement nettement supérieur de 1848 (avec 67 ateliers) [tabl. 2].

\section{Suivi dans le temps des ateliers contemporains : archives cadastrales et communales}

Le dépouillement exhaustif des tableaux indicatifs de sections du cadastre a permis d'assurer un enregistrement exhaustif des établissements tuiliers-briquetiers et de pallier les carences du recensement cartographique. La synthèse des propriétés clôturant les tableaux indicatifs ne fait pas systématiquement apparaitre les ateliers, imposant la consultation exhaustive des tableaux, à l'échelle du canton des Herbiers.

Ce support donne un aperçu du contenu des parcelles. Une récurrence de l'organisation interne des établissements de productions tuilière et briquetière fait apparaître un corpus d'infrastructures caractéristiques (Champagne, 2011). Ces infrastructures comptent une halle de séchage (également désignée sous les appellations galerie ou grange) destinée au stockage et au séchage des produits semi-finis avant cuisson, une unité de cuisson, diverses annexes liées aux usages de la production, ainsi qu'une unité d'habitat domestique à destination de l'artisan-tuilier. Ces infrastructures sont parfois réunies sous la seule appellation tuilerie. Cet ensemble bâti est polarisé autour d'une aire (cour ou ruage), constituant le point de convergence des infrastructures et des étapes de la production (fig. 2) [tabl. 3].

\begin{tabular}{|c|c|}
\hline Supports cartographiques analysés & $\begin{array}{c}\text { Nombre d'ateliers } \\
\text { recensés }\end{array}$ \\
\hline Carte de Cassini (1763-1786) & 10 \\
\hline Carte de l'état-major (1841-1848) & 35 \\
\hline $\begin{array}{c}\text { Plans des cadastres parcellaires } \\
\text { "primitif "/impérial (1808-milieu } \\
\text { du XIx }{ }^{\mathrm{e}} \text { s.) }\end{array}$ & 43 \\
\hline $\begin{array}{c}\text { Tous supports cartographique } \\
\text { confondus }\end{array}$ & 45 \\
\hline
\end{tabular}

Tableau 2 : Nombre d'ateliers recensés dans l'arrondissement de La Rochesur-Yon, selon les supports cartographiques analysés (G. Chamarre).

Table 2: Factories identified in the districts of La Roche-sur-Yon, according the maps analyzes (G. Chamarre).

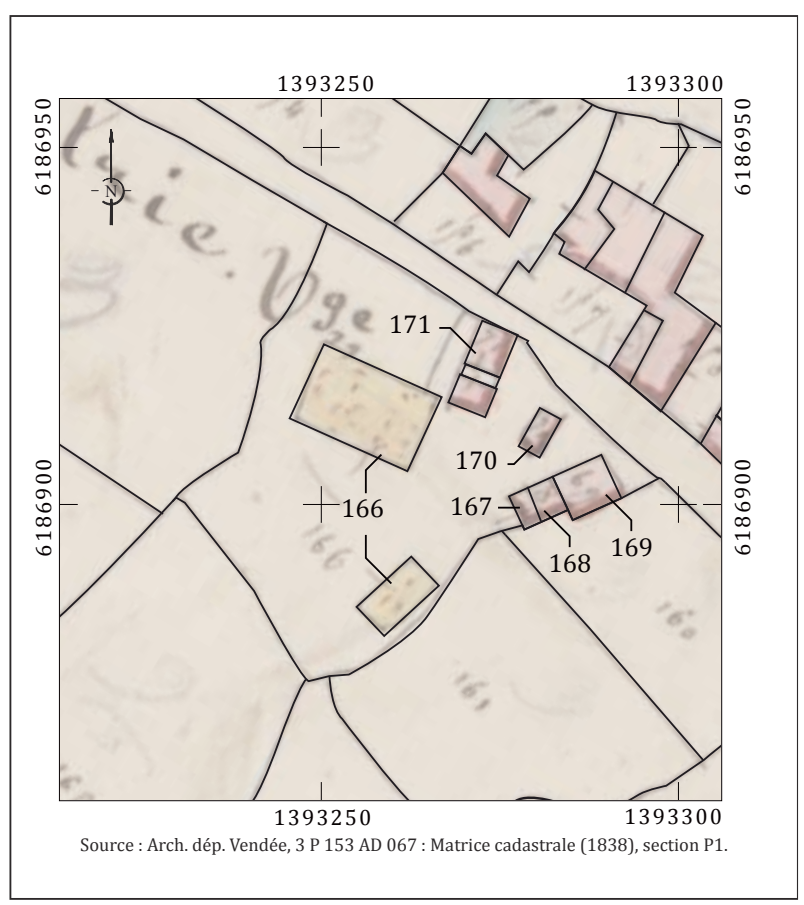

Figure 2 : Plan de la tuilerie de La Poterie de la Jonchère (Mouchamps) extrait du cadastre parcellaire impérial de 1838.

Figure 2: Map of La Poterie de la Jonchère tile-brick factory (Mouchamps) from the imperial land register (1838).

CAD/DAO : G. Chamarre 


\begin{tabular}{|c|c|c|c|}
\hline Noms, prénoms des propriétaires & $\mathrm{N}^{\circ}$ du plan & Désignation particulière de la parcelle & Natures de propriétés \\
\hline Burcerot Louis (1/2) & \multirow{2}{*}{166} & \multirow{2}{*}{ Les halles } & \multirow{2}{*}{ Halles et cour } \\
\hline Burcerot Pierre $(1 / 2)$ & & & \\
\hline Burcerot Louis & 167 & La potrée & Bâtiment \\
\hline Burcerot Louis $(1 / 2)$ & \multirow{2}{*}{168} & \multirow{2}{*}{ Le four commun } & \multirow{2}{*}{ Bâtiment } \\
\hline Burcerot Pierre (1/2) & & & \\
\hline Burcerot Louis & 169 & \multirow{4}{*}{ La potrée } & Bâtiment \\
\hline Burcerot Louis & 170 & & Bâtiment \\
\hline Burcerot Louis $(1 / 2)$ & \multirow{2}{*}{171} & & \multirow{2}{*}{ Sole de four, four à tuiles } \\
\hline Burcerot Pierre (1/2) & & & \\
\hline
\end{tabular}

Tableau 3 : Récapitulatif de la parcelle et du bâti de la tuilerie de La Poterie de la Jonchère d'après le tableau indicatif de section du cadastre de 1838 (G. Chamarre).

Table 3: Overview of the plot and buildings of La Poterie de la Jonchère tile-brick factory (Mouchamps), according to the indicative tables of sections of the cadastre (1838) (G. Chamarre).

Tandis que les tableaux indicatifs de sections proposent un aperçu instantané d'un artisanat, une série d'outils d'imposition offre un suivi dans le temps de ces moyens de production : les matrices des propriétés foncières et les tables d'augmentation ou de diminution d'impositions.

Ces matrices permettent un suivi systématique à la fois des divers changements de propriétaires, remaniements parcellaires et autres faits de constructions ou de démolitions des aménagements non résidentiels, durant le $\mathrm{XIX}^{\mathrm{e}} \mathrm{s}$. jusqu’à 1914.

En appui de ces matrices de propriétés foncières sont jointes les tables d'augmentation et de diminution d'impositions, reprenant les diverses modifications apportées aux infrastructures (construction nouvelle, agrandissement, démolition complète ou partielle). Inhérents à ces tables fiscales, les taux d'imposition ainsi que les années d'application des augmentations ou diminutions sont annotés.

Les projets d'ouverture d'une unité de cuisson artisanale à proximité d'une voie de communication ou d'habitations sont également susceptibles d'avoir fourni des archives. Au même titre qu'un certain nombre de raisons admissibles au rejet d'une demande d'ouverture d'un four vis-à-vis des riverains, tels que les répercutions occasionnées par les émanations insalubres sur les récoltes des parcelles limitrophes, ou encore les risques de propagation d'incendie vers des constructions, bois, forêts ou dépôts permanents de matières sujets à incendie, les unités de cuisson sont désignées comme la source de nombreux inconvénients à l'égard des usagers des axes routiers. Ces inconvénients renvoient aussi bien aux émissions de fumées insalubres enveloppant les voyageurs empruntant l'itinéraire attenant, qu'aux flammes se dégageant au-dessus des fours, susceptibles d'effrayer les animaux de trait et de nuire à la circulation sur les routes les plus proches à la nuit tombée. À ce titre, une enquête des Ponts et
Chaussées se voit prescrite au préalable de chaque ouverture de four à tuiles (Constant, 1881). Les dossiers de demandes d'ouverture de fours (sous-série $5 \mathrm{M}$ « Hygiène et santé ") fournissent des indications sur l'unité de cuisson projetée et son environnement proche, sous la forme de descriptions accompagnées d'un plan détaillé (fig. 3). En l'absence d'un cadre règlementaire précis, un rapport produit en 1856 à la demande de Louis Boudaud, tuilier au village de l'Éraudière (Saint-Fulgent, Vendée), fixe les recommandations suivantes : «[...] la distance minima des tuileries aux routes n’a jamais été déterminée mais [...] il existe une solution pour une question semblable (distance des moulins à vent) dans la dépêche ministérielle du 18 avril 1833 adressée à M. le Préfet de la Vendée. [...] cette dépêche s'en réfère à un ancien arrêt du conseil de l'Artois qui fixe le minimum de distance à 200 pieds pour les routes royales et 150 pieds pour les autres chemins ${ }^{16}$."

En l'absence d'une règlementation adéquate, ces enquêtes n'ont qu'une faible incidence sur les ouvertures de four. En revanche, des compromis prennent régulièrement la forme d'augmentations des projets (levées de terre, haies, maçonneries ou dépendances) préposées à la séparation sanitaire et visuelle de la structure de cuisson avec les riverains et les usagers de la voie carrossable. Les distances, orientations et hauteurs des infrastructures sont de même indiquées. Les rapports permettent ainsi d'appréhender l'organisation spatiale des ateliers et fournissent une date d'ouverture de ces fours.

À une échelle plus locale, un suivi de marchés passés entre les ateliers et les communes peut être évoqué au travers des archives communales et des actes de délibérations municipales produits à l'occasion de la gestion des bâtiments

16. Archives départementales de Vendée, 5M 258/, " Hygiène et santé », commune de Saint-Fulgent, 1833 
Figure 3: Extrait de plan avec représentation d'un projet de construction d'un four à tuiles à la demande d'Alexandre Guichard, tuilier à Mesnard-la-Barotière.

Figure 3: Extract of the map of a building project of a tile-brick factory by Alexandre Guichard in Mesnard-la-Barotière.

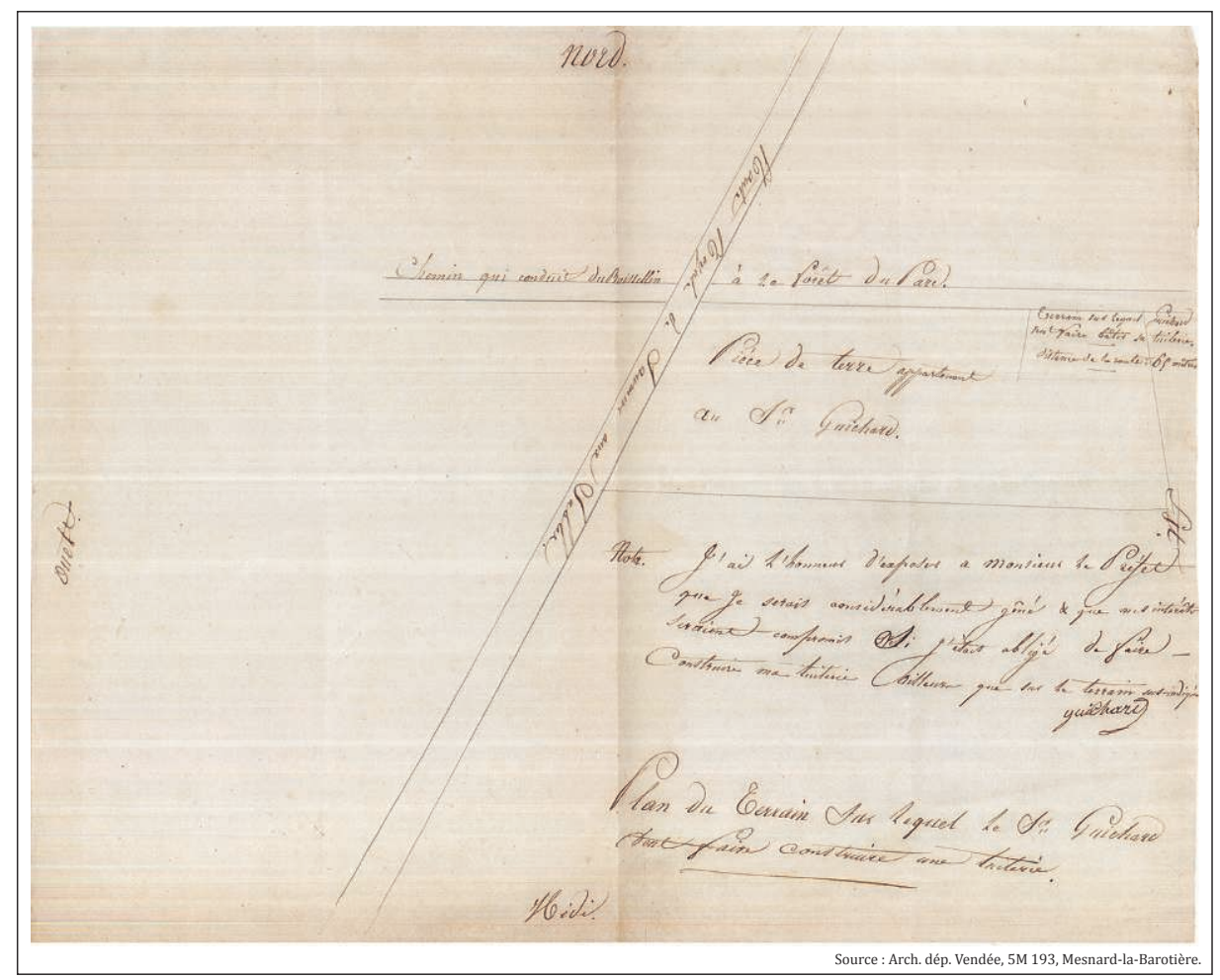

Un formulaire à l'usage des industriels, à raison d'une

et équipements communaux (sous-série $1 \mathrm{O}$ ). Malgré le fait que les fournisseurs de matériaux de maçonnerie et de couverture ne sont que rarement désignés dans les comptes rendus de délibération produits par l'administration, la fourniture des matériaux de construction étant à la charge de l'architecte, quelques exceptions font mention d'ateliers.

Un compte rendu du conseil municipal de Vendrennes, tenu le mardi 19 mars 1839, fait ainsi mention de deux fours à chaux des ateliers respectifs de Blanche-Noue et de Bon[ne]-CEuvre ${ }^{17}$. Sur cette même commune, un autre acte a été fourni à l'occasion d'une séance extraordinaire du conseil municipal, tenue à la date du 21 novembre 1909. L'acte traite d'un défaut de paiement contracté par l'entrepreneur de travaux de couverture du mur de clôture de l'école communale, pour une fourniture de tuiles par le régisseur du four à tuiles de BlancheNoue, Paul Sellier.

\section{Moyens de production et échelles d'exploitation des ateliers : statistiques industrielles}

Les enquêtes agricoles et industrielles destinées à dresser un état numérique des industries sur chaque commune permettent également de mesurer l'importance des productions par établissements à compter du milieu du XIX ${ }^{e} .^{18}$.

17. Archives départementales de Vendée, AC 301/1 : Délibérations municipales

(février 1839-mai 1879), vue 6/75, [en ligne].
18. Archives départementales de Vendée, 6 M 1233-1236 : Statistiques de France fiche par entreprise, fournit à la fois les indications de la commune, la nature de l'établissement. Le nom du propriétaire peut également être indiqué, conjointement à celui du fabricant en charge de l'exploitation de l'entreprise.

Une énumération des objets en circulation dans l'établissement est également relevée : les matières premières employées annuellement selon leur nature (terre à faire tuiles, combustibles, calcaire), leur quantité (en nombre, en kilogrammes, en mètres ou en litres), leurs valeurs unitaire et totale en francs, et selon les lieux d'origine des matières premières (provenance sur les lieux, dans les environs, dans la commune, ou depuis une autre commune énoncée). Les produits fabriqués annuellement sont également inventoriés selon leur nature, leur quantité, leurs valeurs unitaire et totale, et selon les débouchés commerciaux ou lieux de destination des produits.

Un décompte des effectifs et des équipements dont dispose l'établissement est également déclaré : le nombre d'ouvriers et leur statut (hommes, femmes, enfants audessous de 16 ans) ainsi que leurs salaires journaliers différenciés, l'utilisation de moyens mécanisés (moulin à manège à traction par chevaux/mulets, bœufs), ainsi que le type de feux employés (fourneaux, four).

Au milieu du XIx ${ }^{e}$ s., dans le canton des Herbiers, deux stratégies de production apparaissent, en lien étroit avec les capacités de production des ateliers. Ces stratégies proprement dites commerciales correspondent soit à une 
spécialisation de l'offre en tuiles, soit à une diversification de l'offre en différents matériaux.

La spécialisation des productions dans la fabrication de tuiles de couverture semble être le fait des ateliers dont les productions annuelles étaient supérieures à 200000 pièces, atteignant pour certaines les 300000 unités et plus. En termes de débouchés commerciaux, ces productions tuilières se destinent à une diffusion essentiellement locale (fig. 4).

Une exception semble néanmoins s'appliquer aux productions de tuiles en provenance de la commune de Vendrennes, dont la qualité était reconnue bien au-delà du canton. La reconnaissance d'une qualité supérieure d'argile, sur un district de production donné, a ainsi pu donner accès à des débouchés commerciaux sur de plus vastes territoires (aire de diffusion à l'échelle départementale voire régionale) pour des productions exclusivement tuilières.

Une diversification de l'offre en terres cuites architecturales était jusqu'alors restreinte aux ateliers dont les productions annuelles étaient inférieures à 125000 pièces. L'élargissement du panel des produits devait assurer un accès à des débouchés commerciaux géographiquement plus étendus. Jusqu'au milieu du $\mathrm{Xx}^{\mathrm{e}}$ s., les établissements tuiliers-briquetiers du canton des Herbiers semblent avoir été dispensés de diversifier leur production.

Cette diversification tardive de l'offre a été contrainte par une concurrence croissance des productions tuilières de Cholet, La Séginière (Maine-et-Loire), Saint-Martindes-Fontaines (Vendée), ou encore d'Espagne. Parmi les tentatives de diversification de la production artisanale dans le canton des Herbiers, on peut notamment mentionner la production de tuiles à usage ostréicole (tuiles creuses marquées en surface et sous-face par des sillons longitudinaux destinées au captage d'huîtres au stade larvaire) [fig. 5]. Parmi les ateliers concernés, on peut citer la tuilerie de La Jonchère (commune de Mouchamps) dont les productions affluaient jusque dans le marais d'Oléron et le golfe du Morbihan, ainsi que la tuilerie de SaintHubert (commune de Vendrennes) dont les productions affluaient jusqu'à La Trinité-sur-Mer (Morbihan). Ces tentatives d'élargissement de l'offre se révélèrent néanmoins insuffisantes au maintien d'un grand nombre de ces petits ateliers.

\section{Dynamiques d'évolution de l'artisanat tuilier-briquetier à l'époque contemporaine}

La quantification des ateliers à travers le département de la Vendée associée au recensement des ateliers du canton des Herbiers témoignent d'un changement du paysage artisanal tuilier-briquetier de l'extrême fin $\mathrm{du}^{\mathrm{XV}} \mathrm{III}^{\mathrm{e}}{ }^{\mathrm{e}}$ au milieu du $\mathrm{Xx}^{\mathrm{e}} \mathrm{s}$.

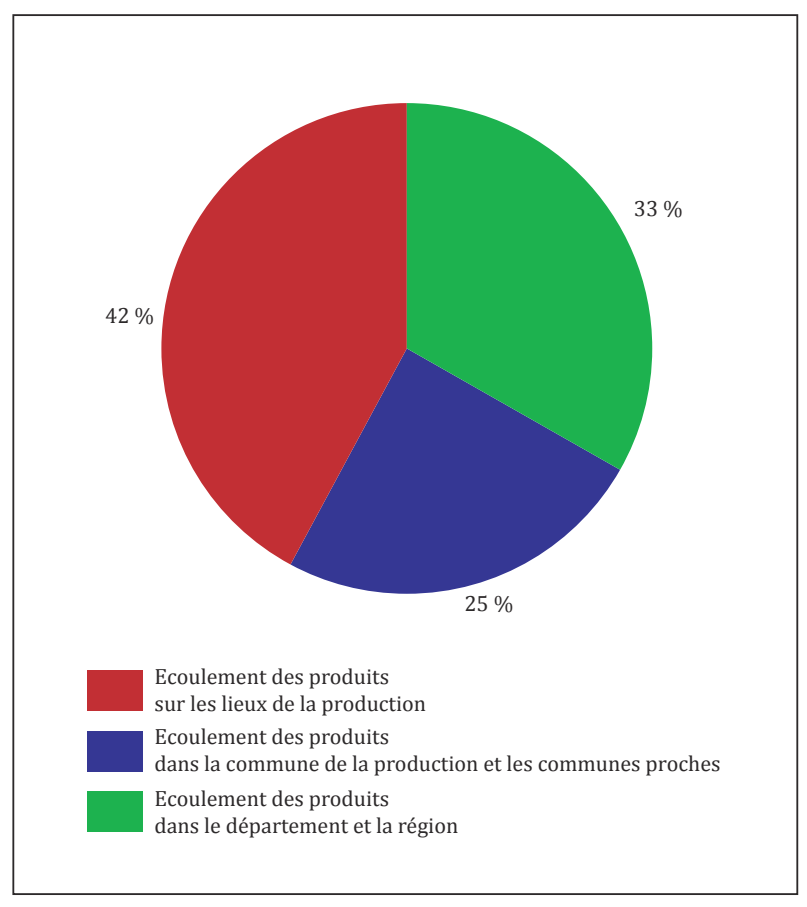

Figure 4 : Représentation des aires de diffusion des productions de terres cuites architecturales provenant du canton des Herbiers, d'après l'état numérique des industries de 1848.

Figure 4: Representation of the sale areas of the ceramics products from the canton of Les Herbiers, according to a list of industries in 1848.

CAD/DAO : G. Chamarre

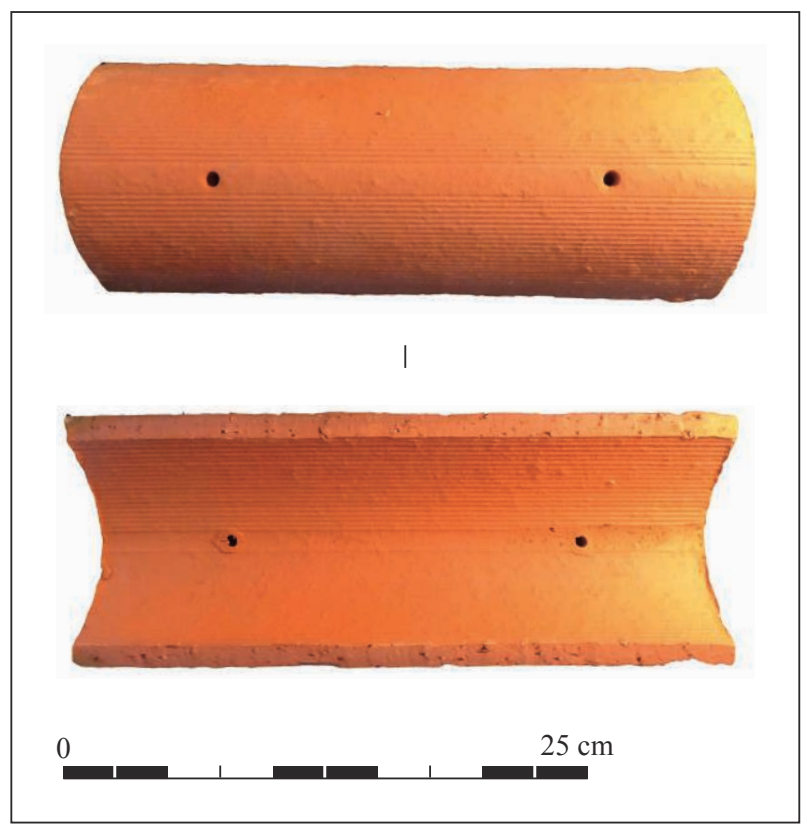

Figure 5 : Tuile creuse ostréicole en terre cuite.

Figure 5: Ceramic oyster tile.

Clichés / Pictures: CAD/DAO : G. Chamarre 
Jusqu'à la fin du XviII ${ }^{e}$ s., l'affiliation d'une production tuilière à un domaine seigneurial ou ecclésiastique semble s'appliquer à l'essentiel des ateliers de tuiliers-briquetiers recensés. Sur le canton des Herbiers, la répartition géographique de six ateliers avérés au XviII ${ }^{\mathrm{e}}$ s. fait apparaître un ensemble de productions tuilières polarisé autour du lieu-dit de La Jonchère. Une localisation de ces ateliers du milieu du XVII ${ }^{e}$ au XVIII ${ }^{e}$ s. reste imprécise en l'absence de cadastre précis.

Au début du XIX ${ }^{e}$ s., une prise d'indépendance de cet artisanat fait apparaître une multitude de petits ateliers répartis uniformément en Vendée (fig. 6). À l'échelle de ce département, le nombre de tuileries est estimé à 120 ateliers, pour une main-d'œuvre de près de 500 individus. Une moyenne annuelle de 6 fournées était conduite, chacune d'elles étant assignée à une production de près de 8000 tuiles, 3000 briques, 300 carreaux et 8 tonneaux de chaux (Cavoleau/de La Fontenelle de Vaudoré, 1844). À l'échelle du canton des Herbiers, la hausse du nombre d'ateliers recensés apparaît avec la fondation de 9 nouveaux ateliers, additionnés aux 5 centres de production avérés au XVIII ${ }^{\mathrm{e}} s$. et maintenus jusqu'au $\mathrm{XIX}^{\mathrm{e}} \mathrm{s}$.

Le milieu du $\mathrm{XIX}^{\mathrm{e}}$ s. constitue la période la plus favorable à l'activité tuilière-briquetière en Vendée. Le département compte jusqu'à 153 tuileries en $1848^{19}$. Au travers des dénombrements des industries tuilières par communes vendéennes, les arrondissements apparaissent inégalement pourvus d'une activité tuilière. Par opposition à une frange littorale de l'arrondissement des Sables-d'Olonne peu fournie en ateliers ${ }^{20}$, une concentration remarquable d'ateliers apparaît nettement circonscrite au canton des Herbiers.

Durant la seconde moitié du XIX ${ }^{e}$ s., un déclin progressif de l'activité tuilière se manifeste par un abaissement du nombre d'ateliers. Ce déclin continu, effectif jusque dans les années 1900, réduit l'artisanat tuilier du canton des Herbiers à six établissements en 1890-189121. Cette part semble se stabiliser à la fin du XIX ${ }^{\mathrm{e}}$ s. et au tout début du $\mathrm{Xx}^{\mathrm{e}} \mathrm{s.}^{22}$.

Un effondrement de l'artisanat tuilier apparaît au travers du dénombrement des tuileries-briqueteries dans le premier quart du $\mathrm{xx}^{\mathrm{e}} \mathrm{s}$. Un témoignage oral recueilli auprès de Fernande Fortin née Rousseau, descendante d'une dynastie locale d'artisans-tuiliers, fait part d'un artisanat désormais réduit à 42 établissements en Vendée

19. Archives départementales de Vendée, 6 M 1233-1236 : Statistiques de France (industrie), situation de fabriques ou manufactures : questionnaires (1848).

20. Les enjeux d'une concurrence avec un centre de production bordelais, dont les matériaux affluaient par la mer, faisaient l'objet de la description de la production tuilière dès 1809 (Cavoleau/de La Fontenelle de Vaudoré, 1844).

21. Les données du dépouillement des annuaires administratifs, statistiques, commerciaux et industriels ont été recueillies auprès de Serge Sellin.

22. ChallLou J. et GRIt E., 1890, Annuaire administratif, statistique, commercial et industriel du département de la Vendée, D. Servant, La Roche-sur-Yon. en $1924^{23}$. Cette diminution brutale du nombre d'établissements de production tuilière coïncide avec un basculement des dynamiques de concurrence. L'artisanat tuilier jusqu'alors exercé par une multitude de petites exploitations familiales aux débouchés économiques locaux est désormais contraint par quelques industries tuilières-briquetières, aux marges de productivité concurrentielles supérieures et aux échelles de diffusion régionales.

Au cours de la seconde moitié du $\mathrm{xx}^{\mathrm{e}}$ s., un maintien relatif du nombre moyen de tuileries comptabilisait 26 ateliers en Vendée et 4 ateliers dans le canton des Herbiers, en activité dans les années 1960 et 1970. Les quelques tentatives de diversification et de compétitivité de l'artisanat tuilier-briquetier sur ce dernier canton n'ont néanmoins pas permis la pérennisation d'une production tuilière à une échelle artisanale.

\section{LA POPULATION TUILIÈRE-BRIQUETIÈRE (FIN XVIII ${ }^{\mathrm{e}}$ S.-DÉBUT XX' ${ }^{\mathrm{E}}$ S.)}

Une observation de l'artisanat au travers de la maind'œuvre est rendue possible grâce aux listes nominatives des recensements quinquennaux de population. Bien que quelques communes disposent de recensements dès l'An IV (1795-1796), ces recensements ne sont systématisés qu'à partir de 1836 à l'ensemble des communes du canton des Herbiers, cela jusqu'en 1911.

Ces listes nominatives recensent les individus par communes et lieux de résidence (quartier, village, hameau, lieu-dit, rue), auxquels sont associés l'âge, la position occupée au sein du ménage d'appartenance, ainsi que la profession. La profession de l'individu constitue de fait le principal élément retenu pour cette étude.

À compter de l'année 1901 et jusqu'en 1911, le statut de l'individu au sein de l'atelier de tuilier est précisé. Un chef d'entreprise est désigné comme patron, tandis qu'un ouvrier se voit associé au nom de l'employeur.

L'intégralité des listes nominatives se référant au canton des Herbiers a été dépouillée. Ce dépouillement s'intéresse alors à une main-d'œuvre tuilière résidente dans les limites du canton, mais potentiellement employée à une activité tuilière à l'extérieur de celui-ci. Afin de s'assurer d'un enregistrement complet de la main-d'œuvre tuilière embauchée dans le canton des Herbiers, potentiellement résidente à l'extérieur de ce canton, le dépouillement a été étendu aux territoires limitrophes des communes ayant porté une production tuilière sur le canton des Herbiers. La recherche d'une main-d'œuvre, employée sur le canton des Herbiers et résidant à l'extérieur de ce dernier,

23. Ce témoignage oral a été consigné par Laurent Blanchard lors d'une enquête orale menée sur le secteur d'Ardelay. 


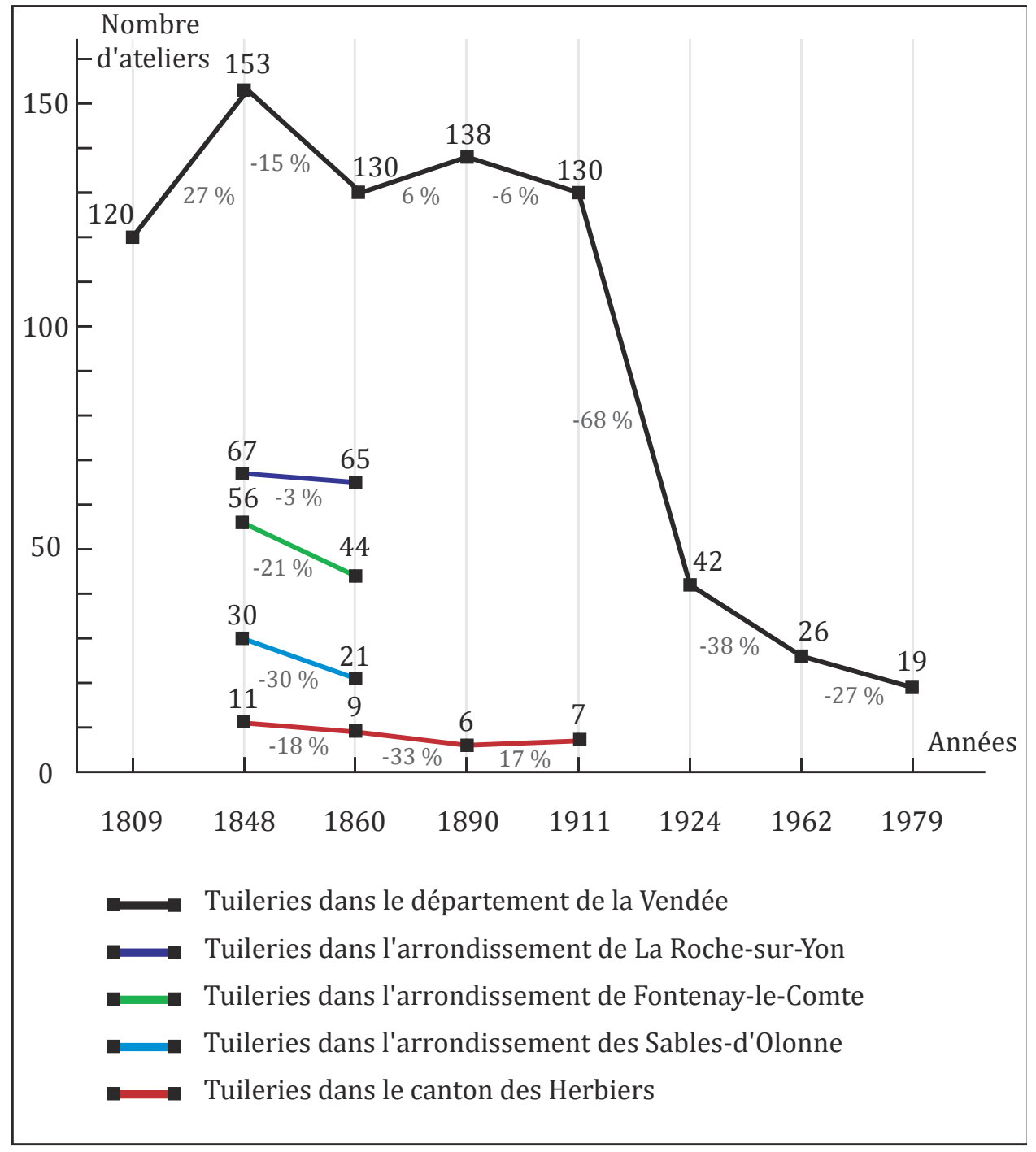

Figure 6 : Évolution comparée du nombre de tuileries-briqueteries du $X I X^{e}$ au $X X^{e}$ S., en Vendée, dans les arrondissements de La Roche-sur-Yon, Fontenay-le-Comte, Les Sablesd'Olonne et dans le canton des Herbiers.

Figure 6: Comparative evolution of the number of tilebrick factories, from 19th to the 2oth century, in Vendée, in the districts of La Rochesur-Yon, Fontenay-le-Comte, Les Sables-d'Olonne and in the canton of Les Herbiers.

CAD/DAO : G. Chamarre

impose de connaître l'employeur et l'atelier de rattachement, réduisant ce dépouillement aux listes nominatives de 1901, 1906 et 1911. Mais aucune correspondance avec une main-d'œuvre résidente à l'extérieur de ce canton n'a été recensée.

Parmi les enjeux sociétaux mis en évidence au travers de ces recensements de population tuilière ont été abordées les questions de la dispersion spatiale et de la mobilité de cette main-d'œuvre au sein du paysage artisanal, les différents individus composant les ateliers et la transmission d'un savoir-faire entre ces membres, prenant parfois la forme de véritables dynasties tuilières.

\section{La dispersion spatiale de la main-d'œuvre tuilière}

La répartition spatiale de la population tuilière coïncide à quelques exceptions près avec la dispersion de cet artisanat sur le canton. Quatre communes ont régulièrement hébergé plus d'un quart de la population tuilière du can- ton et successivement près de la moitié de cette population : Ardelay, Vendrennes, Le Petit-Bourg des Herbiers, puis Mouchamps (tabl. 4).

Ces quatre communes ont reçu des résidents tuiliers en continu, de même pour la commune des Herbiers dans des proportions moindres. En comparaison de ces occupations ininterrompues, la faible représentation d'une population tuilière dans les communes des Epesses et de Mesnard-la-Barotière coïncide avec l'interruption précoce de leurs uniques exploitations tuilières, tandis que les communes de Saint-Mars-la-Réorthe et de Saint-Paulen-Pareds sont dépourvues à la fois d'un artisanat tuilierbriquetier et d'une main-d'œuvre tuilière.

L'artisanat tuilier-briquetier apparaît avoir plus ou moins influé sur la dispersion de l'habitat de la population tuilière-briquetière autour des centres de production.

Sur 42 lieux-dits ayant accueilli des résidents tuiliers sur le canton, près d'un quart est également porteur d'une activité tuilière reconnue. Ramenés à la population tuilière, 


\begin{tabular}{|c|c|c|c|c|c|c|c|c|}
\hline \multirow{2}{*}{ Communes } & \multicolumn{6}{|c|}{ Main-d'œuvre tuilière recensée } \\
\cline { 2 - 10 } & \multicolumn{1}{|c|}{$\mathbf{1 8 3 6}$} & \multicolumn{1}{|c|}{$\mathbf{1 8 5 1}$} & \multicolumn{1}{|c|}{$\mathbf{1 8 8 1}$} & \multicolumn{3}{|c|}{$\mathbf{1 9 0 6}$} \\
\cline { 2 - 10 } & NB & $\%$ & NB & $\%$ & NB & $\%$ & NB & $\%$ \\
\hline Ardelay & 9 & 43 & 8 & 21 & 6 & 23 & 5 & 23 \\
\hline Beaurepaire & 0 & - & 0 & - & 0 & - & 0 & - \\
\hline Les Epesses & 2 & 10 & 0 & - & 0 & - & 0 & - \\
\hline Les Herbiers & 4 & 19 & 8 & 21 & 0 & - & 1 & 5 \\
\hline $\begin{array}{c}\text { Mesnard-la- } \\
\text { Barotière }\end{array}$ & 3 & 14 & 1 & 3 & 1 & 4 & 0 & - \\
\hline Mouchamps & 0 & - & 1 & 3 & 3 & 12 & 10 & 46 \\
\hline $\begin{array}{c}\text { Le Petit- } \\
\text { Bourg-des- } \\
\text { Herbiers }\end{array}$ & 1 & 5 & 4 & 10 & 12 & 46 & 3 & 13 \\
\hline Vendrennes & 2 & 10 & 17 & 44 & 4 & 15 & 3 & 13 \\
\hline
\end{tabular}

Tableau 4 : Représentation de la main-d'œuvre tuilière recensée en 1836, 1851, 1881 et 1906, dans le canton des Herbiers (G. Chamarre).

Table 4: Representation of the tile makers in 1836, 1851, 1881 and 1906, in the canton of Les Herbiers (G. Chamarre).

seuls quinze tuiliers-briquetiers résident au lieu-dit même de l'atelier, lorsque l'essentiel du reste de la population tuilière est réparti sur onze lieux-dits différents, distants de 0,4 à $3 \mathrm{~km}$ des centres de production. Quelques lieux de résidence d'ouvriers tuiliers-briquetiers, au nombre de quatre sont nettement excentrés de plus de $3 \mathrm{~km}$ de l'activité tuilière la plus proche, vraisemblablement attribuables à l'activité tuilière d'une commune limitrophe au canton.

\section{Les dynasties tuilières masculines}

Tout au long du XIX ${ }^{e}$ s., une main-d'œuvre mineure est régulièrement recensée au sein des tuiliers-briqueteries $\mathrm{du}$ canton des Herbiers. Près de 35 ouvriers tuiliers mineurs ont été recensés dans le canton des Herbiers, entre l'An IV (1795-1796) et 1911, parmi lesquels 31 garçons et 4 filles. Tandis que la majorité civile est de 21 ans au $\mathrm{XIX}^{\mathrm{e}}$ s., la main-d'œuvre mineure considérée présente un écart d'âge compris entre 11 ans et 20 ans. L'âge moyen d'embauche des enfants est de 16 ans, tandis que l'implication des individus mineurs à l'activité tuilière s'accroît entre 16 ans et 21 ans. Cette régularité contraste pourtant avec un encadrement législatif, imposé dès la première moitié du XIX ${ }^{e}$ s., mais dont l'artisanat tuilier ne semble que peu pâtir. De même, une sous-estimation de cette main-d'œuvre mineure lors des recensements est plus que probable, notamment au sein des ateliers familiaux.

L'intégration d'un individu mineur dans une activité tuilière peut prendre différentes formes. La professionnalisation résulte majoritairement d'une filiation avec un artisan-tuilier, aussi bien chez les garçons que chez les filles. Sur 35 individus mineurs recensés pour la première fois comme tuiliers entre l'An IV et 1911, dans le canton des Herbiers, 24 sont fils ou filles de tuiliers. Parmi les autres cas observés, l'intégration d'un garçon mineur au sein d'un ménage de tuiliers, en tant qu'ouvrier-tuilier, apparaît à cinq reprises. Un autre cas - cette fois, isolé - correspond à la professionnalisation tuilière d'une fille mineure, à la suite d'un mariage contracté avec un tuilier.

L'ascendance tuilière sur la professionnalisation est moins visible une fois la majorité atteinte. Sur une population masculine de 148 tuiliers recensés dans le canton des Herbiers, entre l'An IV et 1911, la moitié des artisans ne s'inscrit pas dans une activité tuilière familiale. Seul $28 \%$ de cette main-d'œuvre poursuit à l'inverse une activité tuilière familiale, avec 42 artisans identifiés. La main-d'œuvre restante ( $22 \%$ avec 32 individus) présente un patronyme en commun avec une ou plusieurs de la même génération ou d'une génération précédente, sans qu'une filiation puisse être confirmée.

La transmission de la profession de tuilier d'une génération à une autre est majoritairement constatée une fois la majorité de l'individu atteinte et semble concerner, dans une moindre mesure, les individus mineurs, sans pour autant écarter un effet de source. Dans le cas contraire, la participation d'un individu mineur à une activité tuilière donne généralement lieu à une poursuite dans cette activité professionnelle. Toujours sur cette base de 74 tuiliers masculins suggérant une ascendance tuilière, 11 individus ont été recensés comme tuiliers en dessous de la majorité (dont trois ne sont que supposés) (soit 15\%), 49 individus ont été recensés comme tuiliers une fois la majorité atteinte (soit $66 \%$ ), et 14 individus ont été recensés comme tuiliers au-dessous de la majorité puis une fois la majorité atteinte (soit $19 \%$ ).

En l'absence de fils légitime, la transmission masculine de l'activité tuilière peut faire intervenir le gendre tuilier. Cette dépendance de l'atelier envers un gendre se décline successivement par une professionnalisation tuilière $\mathrm{du}$ gendre, par l'incorporation de ce dernier au ménage tuilier, et enfin par la succession à la tête de l'atelier. Cette professionnalisation s'impose dans 7 cas de mariage contracté avec une fille de tuilier sur le canton des Herbiers. Propre au système patriarcal ne permettant pas une transmission filiale de l'activité familiale à une descendance féminine, la fille du maître-tuilier se retrouve désormais reléguée au statut d'épouse de tuilier. La place prédominante du chef de ménage l'emporte donc sur celle de l'épouse ayant légué l'activité tuilière, au profit du gendre. Même si l'absence d'une descendance masculine conduit souvent à une reprise de l'activité par un gendre, ce cas de figure n'est pas systématique. Plusieurs cas de professionnalisation d'un gendre au sein d'une activité tuilière familiale ont ainsi été 
recensés au sein d'ateliers dont la reprise était déjà assurée par une descendance masculine.

\section{La main-d'œuvre tuilière féminine : les filles, épouses et veuves de tuiliers}

Une sous-estimation de la main-d'œuvre tuilière féminine au travers des recensements quinquennaux de population semble accentuée par l'irrégularité de leur dénombrement, suivant les communes et les années de recensement. Au même titre que les statistiques industrielles de $1848^{24}$, ce défaut d'enregistrement des ouvrières tuilières, parfois à cinq ans d'intervalle sur une même commune, ne permet donc pas d'observer une évolution démographique dans le temps.

En comparaison de cet effet de source, la participation de cette main-d'œuvre tuilière féminine dans le fonctionnement des ateliers familiaux, à l'égal de celle des mineur.e.s, apparaît de façon manifeste au travers des enquêtes orales et des documents photographiques (fig. 7). Cette participation apparaît régulièrement attachée au façonnage des produits, à la manutention avant et après cuisson (Orefici et Nauleau, 2019), ou bien encore à la gestion financière de l'atelier comme ce fut le cas pour la tuilerie de Blanche-Noue dans les années 1960 et $1970^{25}$.

La main-d'œuvre tuilière féminine représente 29 filles et femmes tuilières recensées entre 1836 et 1911 dans le canton des Herbiers (contre 143 tuiliers recensés sur la même période), soit une part de $17 \%$ de la population tuilière sur ce canton.

Parmi ce corps ouvrier des femmes-tuilières, apparaissent 3 filles de tuiliers (auxquelles on peut associer 2 autres individus, l'un en raison d'un patronyme commun avec des tuiliers sur ce même canton, l'autre en raison d'une professionnalisation tuilière entretenue dès la petite enfance et partagée avec un frère également mineur), 21 épouses de tuiliers (dont 2 filles de tuilier ayant contracté un mariage avec un tuilier) et enfin 4 veuves de tuiliers ayant repris une activité tuilière (dont seulement une était déjà recensée comme tuilière $\mathrm{du}$ vivant de son époux).

L'implication des femmes de tuiliers dans l'activité de leur époux apparaît forte dans les communes offrant une bonne visibilité à cette main-d'œuvre féminine. Aux Herbiers, 3 des 5 tuiliers mariés ont une épouse tuilière en 1851. Sur la commune du Petit-Bourg-des-Herbiers, 4 des 5 tuiliers mariés ont une épouse tuilière en 1881.

24. Archives départementales de Vendée, 6 M 1234/6 M 1235/6 M 1236 : Statistiques de France (industrie), situation de fabriques ou manufactures : questionnaires (1848).

25. Témoignage de Madeleine et Pierre Rabillard (Blanche-Noue)

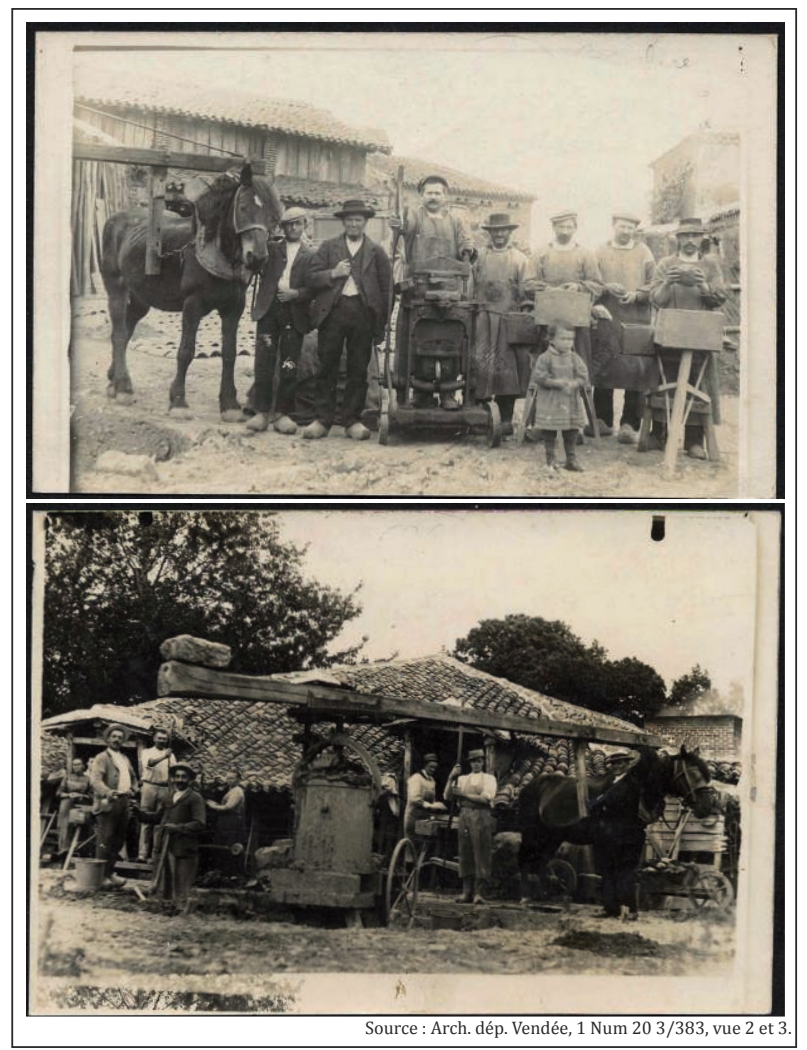

Figure 7 : Cartes postales figurant la tuilerie de La Poterie de la Jonchère (Mouchamps) [en haut] et la tuilerie de Hucheloup (Ardelay) [en bas] en 1920.

Figure 7: Postcards representing La Poterie de la Jonchère tile-brick factory (Mouchamps) (above) and Hucheloup (Ardelay) tile-brick factory (below) in 1920.

Collection J. Meunier

Sur la commune de Vendrennes, 5 des 7 tuiliers mariés ont une épouse tuilière en 1851 , ainsi que 8 des 10 tuiliers mariés ont une épouse tuilière en 1856. La participation des épouses à l'activité tuilière familiale jusqu'à la fin $\mathrm{du} \mathrm{XIX}{ }^{\mathrm{e}}$ s. se répercute sur le rôle des veuves tuilières.

Tandis que le rôle accordé au veuvage dans la perpétuation d'une production tuilière est régulièrement énoncé à propos de l'activité tuilière-briquetière (Moulin, 2011, p. 145), les rôles des veuves au décès de l'époux tuilier suggèrent différents cas de figure.

En l'existence d'un ou plusieurs successeurs masculins (fils ou gendre) en âge de reprendre l'affaire familiale, la veuve peut être démise de ses fonctions à la tête de l'atelier. L'âge moyen pour la reprise d'une activité tuilière par les descendants est estimé autour de la trentaine d'années.

Une deuxième alternative correspond à une veuve de tuilier en charge du ménage et de l'activité tuilière, dans l'attente d'une reprise par l'un des héritiers. Ce cas de figure instauré au décès de l'artisan-tuilier assure une poursuite temporaire de l'exploitation tuilière sous la 
propriété de l'épouse légataire. Ce rôle plus ou moins provisoire donné à la veuve tuilière permet une passation progressive et/ou différée de l'exploitation à une descendance légitime.

Un dernier cas de figure correspond à une veuve de tuilier ayant repris l'activité tuilière au décès de l'époux tuilier, en l'absence de repreneur légitime. Le seul cas dont nous disposons sur le canton des Herbiers témoigne de l'embauche d'un ouvrier tuilier, désormais intégré au ménage sous la tutelle de la veuve tuilière.

Finalement, dès le premier quart $\mathrm{du} \mathrm{xx}^{\mathrm{e}}$ s., une interruption du recensement de la main-d'œuvre tuilière féminine semble coïncider avec un durcissement de la réglementation du travail des femmes au sein des établissements insalubres. Outre le fait qu'une réserve ait temporairement dispensé les " briqueteries en plein air " de certaines contraintes d'embauche, une interruption nette du rôle de la main-d'œuvre tuilière féminine dans les ateliers apparaît peu envisageable. Une interruption du dénombrement des femmes maintenues dans les ateliers semble vraisemblable.

\section{L'APPROCHE ARCHÉOLOGIQUE}

\section{Enregistrement des matériaux de construction contemporains}

L'enregistrement de matériaux de construction produits sur le canton des Herbiers s'est appuyé sur un réseau de collectionneurs de briques et autres matériaux de constructions estampillés, complété par un enregistrement de matériaux en lieu et place de l'atelier de BlancheNoue (commune de Vendrennes).

L'essentiel des données tirées des matériaux de construction contemporains découle des estampilles apposées lors du pressage des modules ou, de manière plus anecdotique, d'inscriptions manuscrites. Les données fournies se classent en trois catégories : une provenance géographique de l'objet (département, commune et/ou lieu-dit), l'indication du nom des producteurs, un millésime.

À l'issue de l'enregistrement, un corpus de 43 briques et 17 tomettes a été enregistré. Parmi ces matériaux, 42 briques et 13 tomettes sont attribués aux $\mathrm{XIX}^{\mathrm{e}}$ et $\mathrm{XX}^{\mathrm{e}} \mathrm{s}$., constituant le principal ensemble de l'enregistrement. Parmi ces matériaux de l'époque contemporaine, 33 cartouches différents ont pu être identifiés (dont 23 sur briques et 10 sur tomettes) [tabl. 5].

L'indication géographique de provenance des matériaux, privilégiant l'annotation du lieu-dit (une part de $70 \%$ ) à celle de la commune (12\%) ou du département (3\%), suggère bien une production à destination d'une demande locale.
À quelques différences près, l'information fournie sur le cartouche d'un même atelier se retrouve à la fois sur les briques et les carreaux. Bien que les changements de formes de cartouches, l'ajout de motifs à une matrice, ou l'adoption de caractères spécifiques apparaissent anecdotiques, d'autres variations dans l'ordre des indications de lieu et de tuilier ou d'orthographe sont en revanche fréquentes. Ces modifications visuelles peuvent constituer autant de moyens de démarcation commerciale des productions entre elles, sur un marché économique local approvisionné par une multitude de petits ateliers.

Parmi des exemples concrets, une distinction des productions associées aux membres de la dynastie tuilière Rousseau, répartis sur différents ateliers distincts, semble avoir été adoptée. Une production estampillée " Rousseau C. " corrobore avec l'exploitation des trois tuileries de Hucheloup, de La Jonchère et de La Poterie de la Jonchère, par Ferdinand Rousseau dès 1904. L'initiale associée au nom du tuilier correspond à l'initiale du nom de l'épouse tuilière, Louise Charbonneau (fig. 8).

L'usage du cartouche dans une démarche commerciale n'est pas un cas isolé, comme permettent de l'attester les tuileries de Blanche-Noue et de Saint-Hubert, sur la commune de Vendrennes. Tandis que l'atelier de BlancheNoue est exploité dès le second quart du XIX ${ }^{e}$ s., la tuilerie voisine de Saint-Hubert n'est construite qu'en 1957-1958. L'exploitation de ce nouvel atelier est alors confiée à un membre de la famille en charge de Blanche-Noue. Dès lors, cette branche de la production bénéficie de l'appellation de Blanche-Noue, pour des raisons commerciales. Le

\begin{tabular}{|c|c|c|c|}
\hline \multicolumn{2}{|c|}{ Données fournies } & \multicolumn{2}{c|}{$\begin{array}{c}\text { Représentation } \\
\text { des indications } \\
\text { estampillées* }\end{array}$} \\
\cline { 3 - 4 } & NR & $\%$ \\
\hline \multirow{2}{*}{$\begin{array}{c}\text { Indications } \\
\text { géogra- } \\
\text { phiques de } \\
\text { provenance }\end{array}$} & Département & 1 & 3 \\
\cline { 2 - 4 } & Commune & 4 & 12 \\
\hline \multirow{3}{*}{$\begin{array}{c}\text { Indications } \\
\text { de l'artisan- } \\
\text { tuilier }\end{array}$} & $\begin{array}{c}\text { Nom dieu-dit } \\
\text { nom de l'artisan-tuilier }\end{array}$ & 23 & 70 \\
\cline { 2 - 4 } & $\begin{array}{c}\text { Initiale de nom de } \\
\text { l'épouse de l'artisan- } \\
\text { tuilier }\end{array}$ & 1 & 73 \\
\hline
\end{tabular}

Tableau 5 : Représentation des indications de lieux de provenance et de producteurs estampillés sur les briques et tomettes pressées du canton des Herbiers (G. Chamarre).

Table 5: Representation of location indications and makers stamped on the briques and tiles from the canton of Les Herbiers (G. Chamarre). * Sur un lot de 33 cartouches (NR) différents enregistrés.

* Sur un lot de 33 cartouches (NR) différents enregistrés 


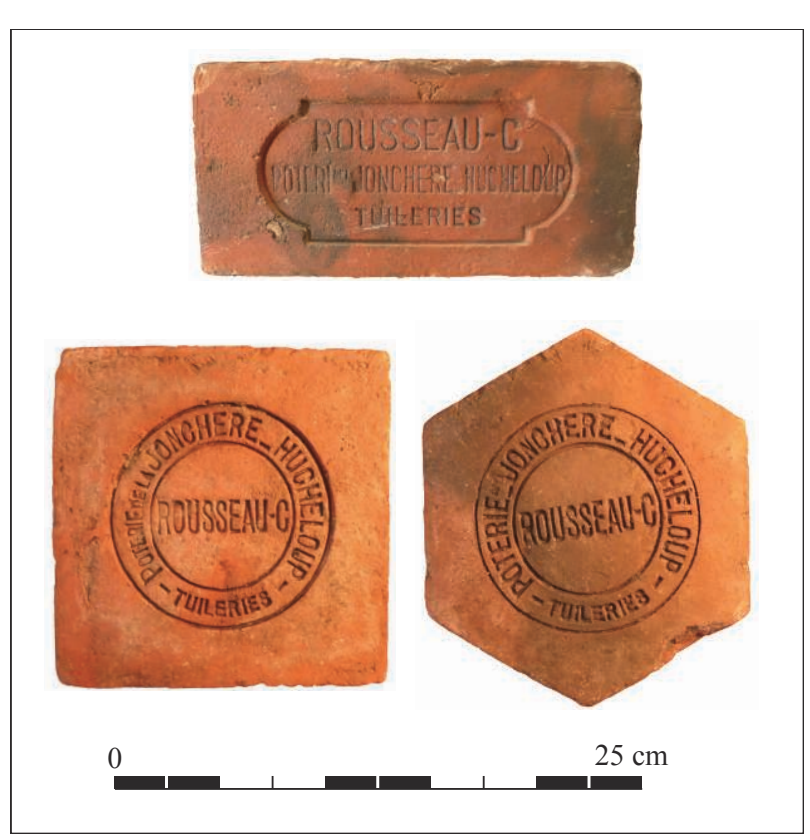

Figure 8: Brique et tomettes pressées provenant des tuileries de La Jonchère (Mouchamps) et Hucheloup (Ardelay) avec indications de noms du tuilier et de son épouse « Rousseau-C[harbonneau]».

Figure 8: Pressed brick and floor tiles from the La Jonchère (Mouchamps) and Hucheloup (Ardelay) factories with names indication of makers (man and woman).

Clichés / Pictures: G. Chamarre

nom de l'exploitation tuilière de Blanche-Noue est alors porteur d'une plus-value, ou du moins d'une reconnaissance, auprès de la demande. Le transfert définitif de la production de Blanche-Noue sur le site de Saint-Hubert, dès 1972, permet la conservation de la main-d'œuvre, désormais associée à cet atelier modernisé ${ }^{26}$.

Un cartouche pourvu d'une indication géographique de provenance et d'un artisan-tuilier permet en principe une localisation précise de l'atelier de production ainsi qu'une estimation chronologique en appui des sources archivistiques.

Inversement, l'apposition d'un millésime reste peu fréquente, voire tout simplement absente sur les cartouches pressés issus du canton des Herbiers. L'annotation manuscrite de millésimes apparait quant à elle régulière.

Un cas particulièrement évocateur correspond à un lot de quatre carreaux de pavement, mis au jour à la Fretière d'Ardelay et portant des inscriptions manuscrites dans l'argile avant cuisson. Trois de ces carreaux fournissent le même millésime, renvoyant à l'année 1747. Issus vraisemblablement d'un seul et même atelier, établi au lieu-dit de La Ganachère, paroisse d'Ardelay, les noms de deux artisans-tuiliers sont annotés. Ils sont respectivement dénommés Alain Brüin et Jean Dupuyt (fig. 9). Le caractère non

26. Témoignage de Madeleine et Pierre Rabillard (Blanche-Noue) conventionnel de ces inscriptions semble avoir détourné ces pièces d'une pose au sol, comme peut en témoigner le contexte de découverte de ces dernières, entreposées dans un grenier. Actuellement, ce lot de carreaux est conservé dans les collections archéologiques de l'Historial de Vendée (Les Lucs-sur-Boulogne). Tandis qu'aucune autre mention n'atteste d'une activité tuilière au lieu-dit de La Ganachère tout au long du XviII ${ }^{e}$ s., le maintien de cet artisanat est suggéré par un devis prévisionnel des réparations sur le presbytère de la commune de Saint-Mars-la-Réorthe. Ces réparations incluent une réfection de la couverture, nécessitant une fourniture de 600 tuiles. Daté du $1^{\text {er jan- }}$ vier 1811, ce devis émane d'un dénommé Guaidon, tuilier à La Ganachère ${ }^{27}$. Un seul artisan-tuilier recensé au XIX ${ }^{e} s$. sur le canton des Herbiers peut lui être associé, recensé sous le nom de Louis Guesdon et résidant en 1817 sur la commune du Petit-Bourg-des-Herbiers ${ }^{28}$. L'absence définitive de ce patronyme à une date ultérieure ou d'un artisan-tuilier résidant à La Ganachère laisse supposer une interruption de cette activité dans les années suivantes.

De semblables inscriptions manuscrites apparaissent également lors de la dépose de toitures anciennes ou au sein même des ateliers de production.

La réfection de la couverture de la grange orientale de l'établissement tuilier de Blanche-Noue (commune de Vendrennes) a ainsi révélé une tuile creuse portant le millésime « $1846 »^{29}$.

L'intérêt d'un enregistrement des cartouches en contexte archéologique, au sein des collections privées et dans les ateliers disposant d'échantillons de leur production semble propre à documenter un artisanat local. À plus long terme, de semblables bases de données devraient permettre une datation de contextes archéologiques parfois à quelques années près. Ce biais de datation est d'autant plus appréciable à l'égard des inscriptions manuscrites, régulièrement datées ${ }^{30}$.

\section{Enregistrement de terrain et sauvegarde des données archéologiques}

Le recensement des tuileries-briqueteries contemporaines dans le seul canton des Herbiers souligne la préservation d'une infime part des ateliers encore en élévation. Complémentaire d'une compréhension de l'évolution de

27. Archives départementales de Vendée, 10717 : Édifices du culte : église et presbytère (1809-1854).

28. Archives départementales de Vendée, 6 M 275 : Recensement de population, Petit-Bourg-des-Herbiers (Le) (1817), vue 15/15.

29. Cette tuile est actuellement conservée par les propriétaires du site.

30. L'intérêt d'une étude des tuiles inscrites par une approche typologique et anthropologique est également souligné sur d'autres secteurs géographiques, comme cela est actuellement proposé en Argonne (départements de la Marne et de la Meuse) par Colette Mechin (université de Strasbourg, UMR 7363 CNRS \& Unistra « SAGE »). 


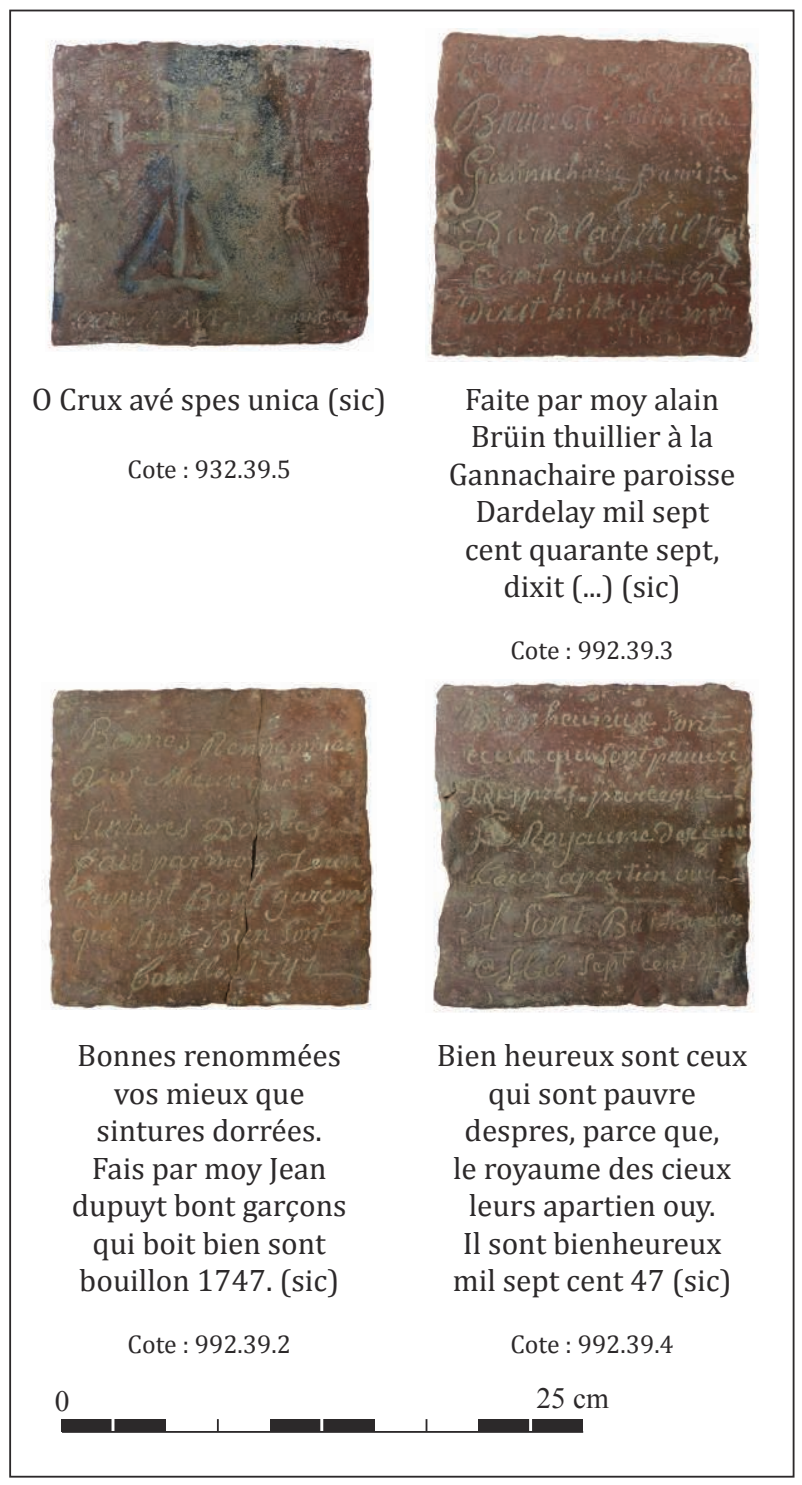

Figure 9: Carreaux manuscrits provenant de La Ganachère (Ardelay).

Figure 9: Handwritten floor tiles from La Ganachère of Ardelay (Vendée).

Clichés / Pictures: G. Chamarre

ces exploitations et de la main-d'œuvre associée, l'enregistrement archéologique de ces infrastructures artisanales apparaît nécessaire.

L'enregistrement du four à tuiles de Blanche-Noue (Vendrennes) a ainsi été entrepris. La nécessité d'une méthodologie d'enregistrement du bâti applicable sur un temps succinct sur le terrain et un corpus étendu de fours s'est conclue par l'application d'un protocole d'enregistrement appuyé d'un croquis et des observations suivantes : une mesure exhaustive des volumes, un enregistrement de cotes de triangulation afin de pallier les déformations des espaces et d'assurer un rendu fiable des dimensions $\mathrm{du}$ bâti, un relevé des maçonneries complexes, ainsi qu'une identification des matériaux de construction employés et des anomalies structurelles (réaménagements, réfections, comblement, etc.).

Un enregistrement préventif des infrastructures de production est d'autant plus nécessaire qu'un certain nombre d'établissements ont été progressivement supprimés depuis l'arrêt de leur activité. À l'issue du recensement cartographique effectué sur l'arrondissement de La Rochesur-Yon, le constat était éloquent : près de la moitié des tuileries-briqueteries recensées (une part de $52 \%$ ) avait été supprimée pour la plupart à l'interruption même de leur exploitation tuilière. $24 \%$ de sites étaient positionnés sur d'actuelles parcelles agraires et $17 \%$ en contexte forestier, contre seulement $7 \%$ des sites ayant conservé des infrastructures de production en élévation (fig. 10).

\section{Relevé bâti du four à tuiles de Blanche-Noue}

L'implantation d'une activité tuilière au lieu-dit de Blanche-Noue, en lisière occidentale du parc de la forêt de Soubise, peut suggérer le déplacement d'une production tuilière initialement localisée sur le site de l'" Ancienne tuilerie de la forêt » sous la propriété de la famille de Chabot. Ce transfert d'activité aurait été encouragé par la recherche de débouchés commerciaux avantageux. L'isolement de l'ancien site tuilier, implanté en lisière du domaine forestier durant le dernier quart du XviII ${ }^{\mathrm{e}}$ s. et éloigné de près de $2 \mathrm{~km}$ de la Route Royale $\mathrm{n}^{\circ} 160$ reliant Les Herbiers à Vendrennes, aurait ainsi été supplanté au profit du site de Blanche-Noue situé à $620 \mathrm{~m}$ de l'axe routier stratégique.

Une activité tuilière sur le site de Blanche-Noue est avérée en 1838, au travers du cadastre parcellaire impérial. À cette date, la tuilerie appartient au propriétaire du château du parc Soubise, Alexandre de Chabot. Le site de Blanche-Noue est successivement transmis à Auguste de Chabot puis à Guillaume de Chabot en $1882^{31}$.

Le four de la tuilerie contemporaine de Blanche-Noue a été construit en 1904, d'après les millésimes manuscrits figurant sur des briques intégrées à la maçonnerie de façade : "BLANCHENOUE. Ce four a été construit en 1904, par Louis Moreau, régisseur BERTELOT, maçon ». Une autre inscription fait cette fois mention du propriétaire de l'atelier : «M. le Comte de Chabot ». Cette unité de cuisson correspond néanmoins à un réaménagement des infrastructures de production, une activité tuilière au lieu-dit de Blanche-Noue étant attestée dès 1838 au travers du cadastre napoléonien ${ }^{32}$. La tuilerie appartient alors au propriétaire du château du parc Soubise, Alexandre

31. Archives départementales de Vendée, 3 P 3380 : Table alphabétique des propriétaires (1839-1914), vol. unique, fo 129/720.

32. Archives départementales de Vendée, 3P 301 CE 015 : Cadastre parcellaire impérial (1838), section D4. 


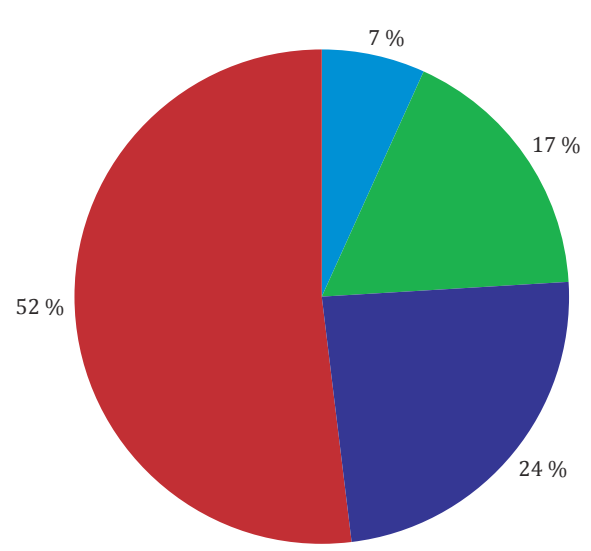

Sites anciennement démolis en fin d'exploitation

Sites actuellement positionnés en contexte agraire

Sites actuellement positionnés en contexte forestier

Sites ayant conservés des infrastructures en élévation

Figure 10 : Représentation des sites de production tuilière-briquetière recensés dans l'arrondissement de La Roche-sur-Yon selon leurs états de préservation et leurs milieux d'enfouissement.

Figure 10: Representation of tile-brick factories, from $19^{\text {th }}$ to $20^{\text {th }}$ century, in the districts of La Roche-sur-Yon, according to their preservation and burials environments.

\section{CAD/DAO : G. Chamarre}

de Chabot $^{33}$. Cette annotation apparaît comme l'une des seules mentions connues pour la première moitié du $\mathrm{XIX}^{\mathrm{e}} s^{34}$ (fig. 11).

Il s'agit d'un four couché d'une longueur de 11,5 m et d'une largeur de $6 \mathrm{~m}$. La hauteur du four est de $3 \mathrm{~m}$, surmonté d'une cheminée atteignant $6 \mathrm{~m}$ au-dessus du niveau du sol (fig. 12 et 13).

Le parement externe est constitué d'une maçonnerie de blocs de schiste liés par du mortier, avec des chaînages d'angles associant blocs de granite et de schiste. Des matériaux de construction en terre cuite sont employés à la couverture du four (tuiles creuses), à l'élévation de la cheminée (briques creuses), à la maçonnerie interne de la chambre de cuisson ainsi qu'à l'encadrement des ouvertures (briques pleines). L'épaisseur des parois varie entre 1,2 et $1,5 \mathrm{~m}$, soutenue au nord par l'ajout de renforts en béton moulé avec la date inscrite " 1958 ».

Le chargement de la chambre de cuisson était procédé depuis la façade nord. Le percement de regards de part

33. Archives départementales de Vendée, 3 P 3378 : Tableaux indicatifs (1839), section D4, vue 74/216.

34. Cette tuile millésimée a été mise au jour sur la toiture de la grange orientale de l'établissement de Blanche-Noue. Elle est actuellement conservée par les propriétaires du site.

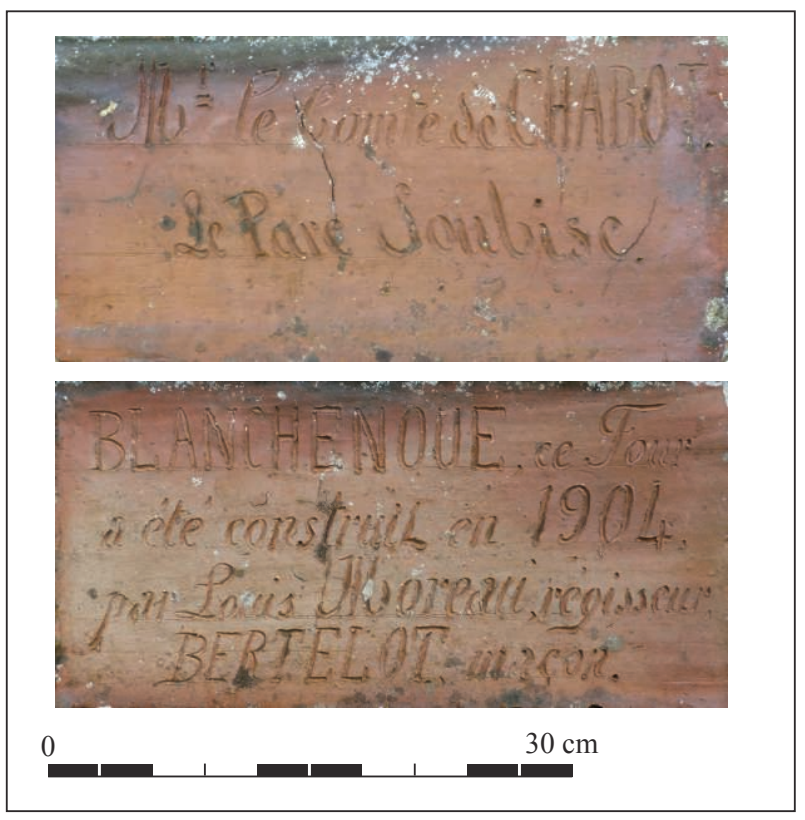

Figure 11 : Briques avec inscription « BLANCHENOUE. Ce four a été construit en 1904, par Louis Moreau, régisseur BERTELOT, maçon » provenant de la façade nord du four à tuiles de Blanche-Noue.

Figure 11: Handwritten bricks from the front of the oven of the BlancheNoue tile-brick factory.

Clichés / Pictures : G. Chamarre

et d'autre de l'ouverture d'enfournement permettait de surveiller la progression des phases de cuisson du chargement, tandis que l'accès principal est temporairement cloisonné lors du fonctionnement du four.

La chambre de cuisson couvre $22,63 \mathrm{~m}^{2}$ pour un volume d'enfournage de $3170 \mathrm{~m}^{3}$. L'appareillage des parois est constitué de briques disposées en opus testaceum et se poursuit dans la voûte surbaissée d'une hauteur de 1,21 m puis la voûte en cul-de-four au-dessus de l'alandier au sud. La paroi orientale du four présente les indices de rebouchage d'un autre accès de chargement. Ce rebouchage serait antérieur à la reprise de l'exploitation en 1946 par les frères Gaston Blanchard et Florent Blanchard ${ }^{35}$. L'extrémité sud de l'alandier débouche sur la gueule du foyer, par laquelle était alimenté le foyer. L'arche de cette ouverture est composée de gros appareils en pyrophyllite. Cette roche réfractaire d'origine métamorphique est communément employée sur les fours à chaux de la région. Un affleurement de cette roche a été exploité sur le site de La Pierre des Plochères, dans la commune de Saint-Pierre-du-Chemin (Vendée) [Viaud, 2010]. L'alandier surplombe quant à lui le cendrier aménagé en partie souterraine, séparé par une voûte ajourée en briques permettant l'évacuation des cendres. Le tirage de ces cendres était effectué depuis une trappe latérale accessible d'une loge extérieure aménagée en contrebas du four.

35. Témoignage de Madeleine et Pierre Rabillard (Blanche-Noue). 


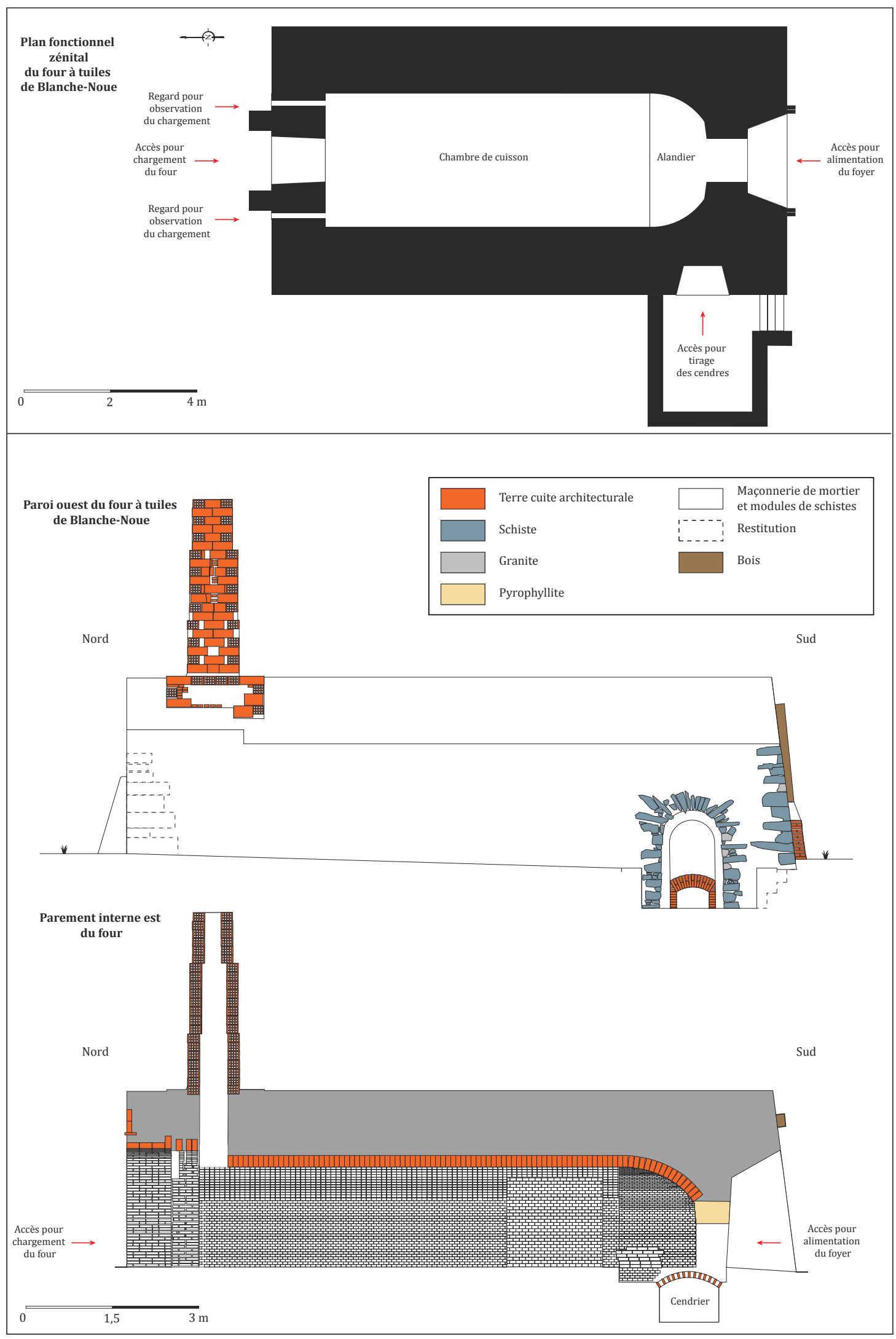

Figure 12 : Plan fonctionnel et relevés du four à tuiles de Blanche-Noue (Vendrennes) [1/2].

Figure 12: Record of the Blanche-Noue tiles oven (Vendrennes) [1/2]. 


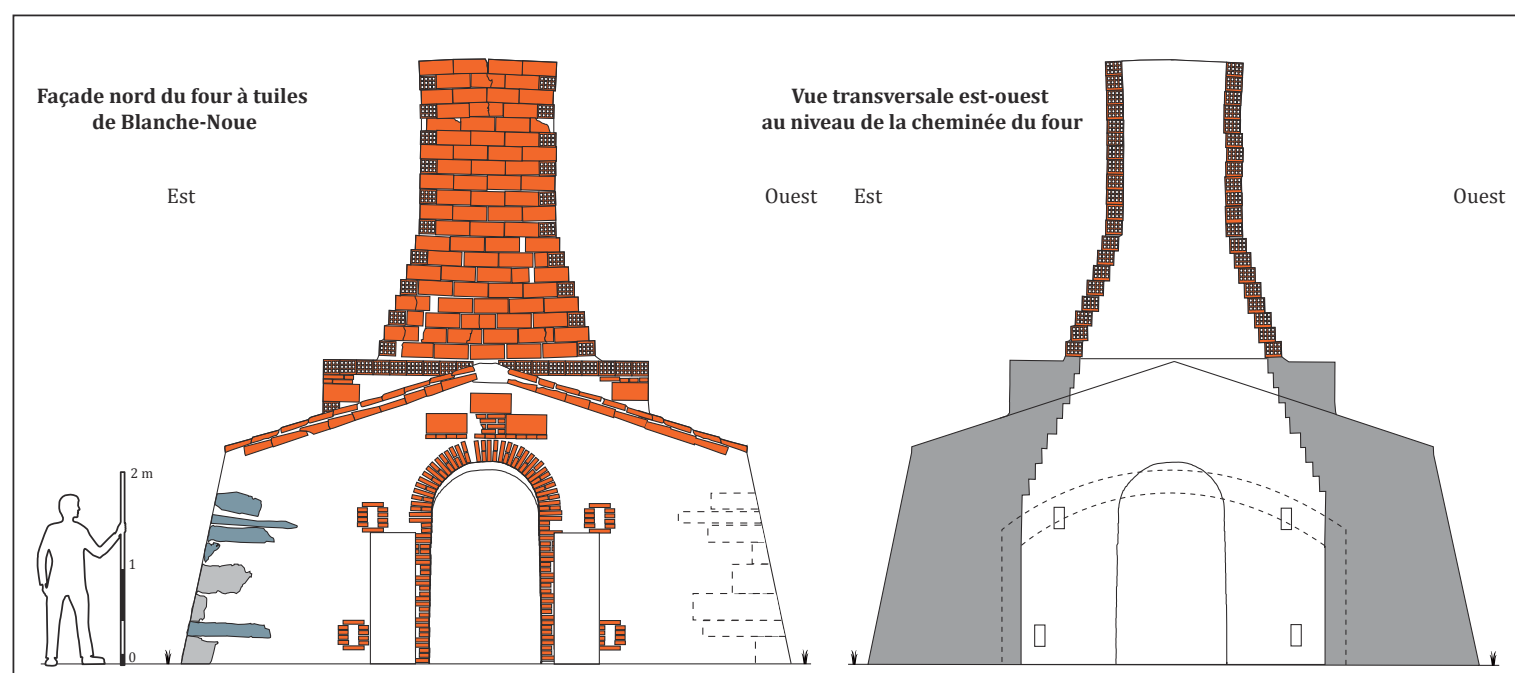

Façade sud du four à tuiles de Blanche-Noue

Vue transversale ouest-est au niveau de l'alandier et du cendrier du four

Vue zénitale de la voûte du cendrier du four

0 1,5 $3 \mathrm{~m}$

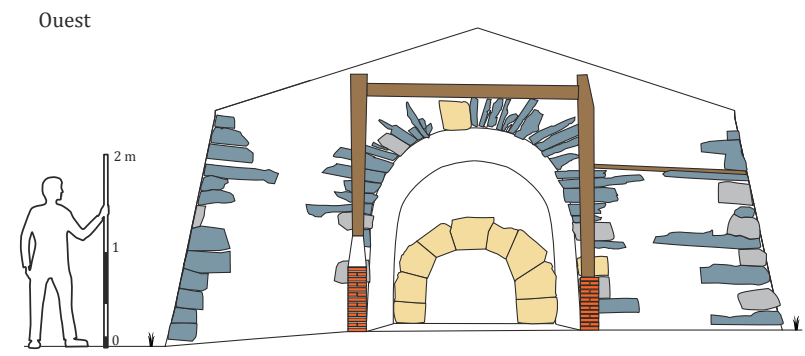

Est
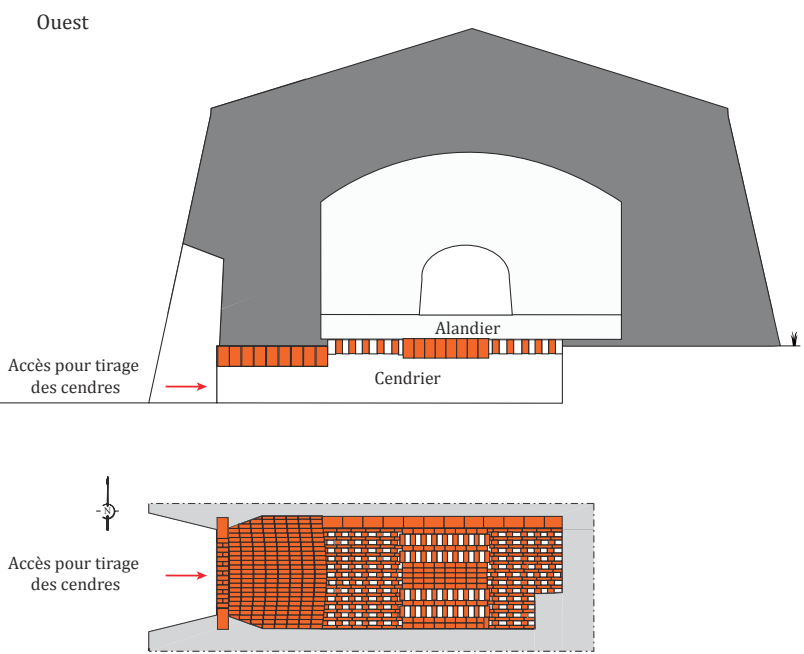

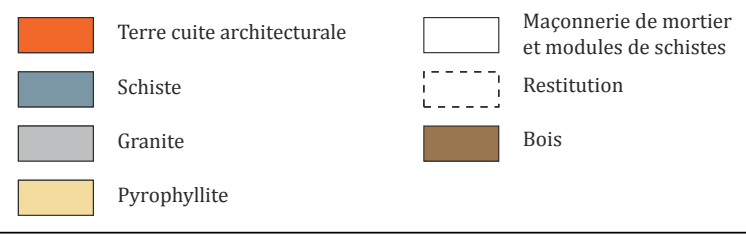

Figure 13: Relevés du four à tuiles de Blanche-Noue (Vendrennes) [2/2].

Figure 13: Record of the Blanche-Noue tiles oven (Vendrennes) [2/2]. 
Lors de l'enfournement, les premiers mètres au contact de l'alandier étaient occupés par les briquaux et la pierre calcaire destinée à produire de la chaux. La majeure partie du volume d'enfournage était occupée par les briques et tuiles, tandis que l'espace à l'opposé de l'alandier était réservé aux briques plâtrières et briques à plafonds. Les matériaux étaient disposés sur toute la hauteur sous voûte, avec le maintien de dix couloirs de circulation de la chaleur entre les marchandises. Au défournement, la contenance du four s'élevait entre 18 et 20 tonnes de marchandises ${ }^{36}$.

\section{CONCLUSION}

L'étude diachronique menée sur l'artisanat tuilier-briquetier du canton des Herbiers a permis de tirer les enseignements méthodologiques quant à l'application d'une étude archivistique à propos de l'artisanat des terres cuites architecturales du XIX ${ }^{e} s$.

Le recensement des ateliers apparaît comme le principal aboutissant de l'étude, avec près de 16 établissements recensés entre le $\mathrm{XvII}^{\mathrm{e}}$ et le $\mathrm{Xx}^{\mathrm{e}}$ s. sur le canton. L'essentiel du corpus a été tiré des tableaux indicatifs de sections cadastrales. Certains centres de production sont signalés dès l'époque moderne au travers des fonds seigneuriaux et ecclésiastiques.

Jusqu'à la fin du Xvini ${ }^{\mathrm{e}}$ s., l'affiliation d'une production tuilière à un domaine seigneurial ou ecclésiastique semble s'appliquer à l'essentiel des ateliers tuiliers recensés. Néanmoins, les fonds seigneuriaux et ecclésiastiques ne font aucune mention des ateliers indépendants des grands domaines. Cet effet de source laisse échapper toute une frange de l'artisanat tuilier dont l'existence n'est renseignée que par de rares indices, à l'égal d'une production de carreaux inscrits provenant de La Ganachère d'Ardelay. Dans le courant du $\mathrm{XIX}^{\mathrm{e}}$ s., la multiplication par deux du nombre d'ateliers recensés sur le canton des Herbiers semble témoigner d'une appropriation des moyens de production par la population artisanale indépendante, faisant apparaître une multitude de petits ateliers, répartis de façon homogène sur le territoire. Un district de production tuilière apparait clairement autour du lieu-dit de La Jonchère. La pérennité de la tradition tuilière sur un district circonscrit souligne le fort potentiel archéologique de celui-ci. Le paysage artisanal tuilier, représenté par une multitude d'ateliers familiaux, semble se maintenir jusqu'au début du xx $x^{e}$ s. L'effondrement de cet artisanat dans le canton des Herbiers correspond à l'implantation d'industries tuilières-briquetières régionales.

Une compréhension de l'organisation des infrastructures de productions tuilières au sein des ateliers transpa-

36. Témoignage de Madeleine et Pierre Rabillard (Blanche-Noue) raît au travers des relevés planimétriques des cadastres et des tableaux indicatifs de sections associés. Les matrices des propriétés foncières puis des propriétés non-bâties, documentent quant à elles les remaniements à la fois bâtis et non-bâtis. L'aspect évolutif de ces matrices de propriétés permet un suivi des divers changements de propriétaires, et autres faits de réaménagements, constructions ou démolitions. Ces outils d'imposition font apparaittre les propriétaires, ignorant les éventuels usufruitiers en charge de l'exploitation des ateliers.

Une approche davantage microéconomique est suggérée au travers de certaines enquêtes industrielles du $\mathrm{XIX}^{\mathrm{e}} \mathrm{s}$. Aux ateliers se voient associés des volumes de production et leurs échelles de diffusion, ainsi que les moyens mécaniques ou humains dont ces ateliers disposent. Outre une approche économique et sociale associée à ces ateliers, l'exploitant est annoncé distinctement du propriétaire.

À l'échelle de l'atelier, une organisation sociale traditionnelle tend à dissocier plusieurs membres inhérents de ce corps ouvrier. L'exploitation est placée sous l'autorité des tuiliers, suivis des épouses et veuves tuilières. À plus long terme, la femme tuilière est garante de la pérennité de l'activité familiale. La participation des enfants tuiliers est également constante dans le temps. La plupart de ces enfants appartiennent à des dynasties tuilières.

Parmi les autres moyens d'investigation testés lors de cette étude, l'enregistrement des matériaux se révéla peu concluant à court terme. Le recoupement des inscriptions sur cartouches et des archives permet une appréhension quasi systématique des provenances et datations de ces matériaux de construction. À plus long terme, l'enrichissement d'une base de données de matériaux de construction devrait trouver un cas d'application concret lors de futures études de bâtis du XIX ${ }^{\mathrm{e}}$ s. Les matériaux employés dans ces constructions devraient ainsi fournir des éléments de datation évidents.

Enfin, en appui d'un relevé bâti des infrastructures de production, les enquêtes orales auprès d'anciens artisans tuiliers permettent de comprendre à la fois les infrastructures, les gestes et les productions qui leur sont associés. L'accès à ce témoignage oral et aux infrastructures bâties est néanmoins tributaire du temps et mérite que l'on s'y intéresse dans les prochaines années.

\section{Bibliographie}

Arnoux M., 2013, « Districts industriels, régions de production, marchés (Europe, $\mathrm{XII}^{\mathrm{e}}-\mathrm{XV}^{\mathrm{e}} \mathrm{s}$.). Quelques pistes de réflexion ", dans Minovez J.-M., Verna C. et Hilaire-Pérez L. (dir.), Les industries rurales dans l'Europe médiévale et moderne, Toulouse, Presses universitaires du Mirail, p. 13-28. 
Cavoleau J.-A. et de La Fontenelle de Vaudoré A.-D., 1844, Statistique ou description générale du département de la Vendée, Paris, Librairie Dumoulin, 945 p.

Chamarre G., 2018, L'artisanat tuilier-briquetier : état des connaissances et recensement des ateliers dans l'arrondissement de La Roche-sur-Yon (Vendée), aux XVIII et XIX s., d'après les sources écrites, mémoire de master 1 , archéologie moderne et contemporaine, université de Nantes sous la direction d'Yves Henigfeld, 2 vol.

Chamarre G., 2019, L'artisanat tuilier-briquetier dans le canton des Herbiers (Vendée) : perspectives de recherches archéologiques et protocole d'étude appliqué à un artisanat tuilier contemporain, mémoire de master 2 , archéologie moderne et contemporaine, université de Nantes sous la direction d'Yves Henigfeld, 1 vol.

Champagne A., 2007, L'artisanat rural du Haut-Poitou, Rennes, Presses universitaires de Rennes, 244 p.

Champagne A., 2011, "L'apport des sources écrites à la connaissance des ateliers de production de terres cuites poitevins (XIV"-XVIII ${ }^{\mathrm{e}}$ s.) ", dans BoCQUET-LIÉNARD A. et FAJAL B. (dir.), À propo[t]s de l'usage, de la production et de la circulation des terres cuites dans l'Europe du Nord-Ouest (XIV-XVI s.), Caen, Publications du CRAHM, p. 155-166.

Chapelot J., Сhapelot O. et Rieth B. (dir.), 2009, Terres cuites architecturales médiévales et modernes, Caen, Publications du CRAHM, 456 p.

Constant C., 1881, Code des établissements industriels classés ateliers dangereux, insalubres ou incommodes, Paris, A. Durand et Pedone-Lauriel, $312 \mathrm{p}$.

Costa L. et Robert S. (dir.), 2008, Guide de lecture des cartes anciennes, Paris, Errance, 104 p.

Gabilly J., 1978, Poitou, Vendée, Charentes, Paris, Masson, 199 p.

Goujou J.-C., Debrand-Passard S., Hantzpergue P. et
Lebret P., 1994, Notice explicative, Carte géologique France (1/50000), feuille Les Sables-d'Olonne - Longeville (584), Orléans, BRGM, 95 p.

Longnon A., 1922, Les noms de lieu de la France, Paris, Honoré Champion, $831 \mathrm{p}$.

Minovez J.-M., Verna C. et Hilaire-Pérez L. (dir.), 2013, Les industries rurales dans l'Europe médiévale et moderne, Toulouse, Presses universitaires du Mirail, $310 \mathrm{p}$.

Moulin M.-A., 2011, "Tuileries, tuiliers et tuiles de la région d'Argentan à la fin du Moyen Âge d'après les sources écrites ", dans Bocquet-Liénard A. et Fajal B. (dir.), $\grave{A}$ propo[t]s de l'usage, de la production et de la circulation des terres cuites dans l'Europe du Nord-Ouest (XIV'-XVI s.), Caen, Publications du CRAHM, p. 137-153.

Orefici L. et Nauleau J.-F., 2019, « Les tuileries d'Arthon (Chaumes-en-Retz, Loire-Atlantique) : un site majeur à découvrir ", dans Thuillier F. (dir.), Les terres cuites architecturales en France du Moyen âge à l'époque contemporaine: Recherches sur les tuileries et les productions tuilières, Archéologie Moderne et Contemporaine, 6, Drémil Lafage, Éditions Mergoil, p. 299-334.

Rézeau P., 2003, Premier dictionnaire du Patois de la Vendée, La Roche-sur-Yon, Centre vendéen de recherches historiques, $352 \mathrm{p}$.

Viaud J.-M., 2010, «Fiche argumentaire de présélection des sites : inventaire du patrimoine géologique des Pays-dela-Loire », BRGM Pays-de-la-Loire, [En ligne, consulté le 13/05/2019 : http://www.donnees.pays-de-la-loire. developpement-durable.gouv.fr/data/fiches/site_preselectionne_inpg/IPG85_FA007.pdf].

Vue B., 2009, "Localiser et reconnaître un site de tuilerie : l'exemple de la Haute-Marne ", dans CHapelot J., ChapeLOT O. et RiETH B., Terres cuites architecturales médiévales et modernes, Caen, Publications du CRAHM, p. 53-61. 


\title{
Das Ziegeleihandwerk im Bereich des Kantons „Les Herbiers“ in der Vendée: methodisches Vorgehen und Forschungsperspektiven
}

Dieser Artikel präsentiert die wesentlichen Ergebnisse einer Studie, die im Rahmen eines zweijährigen Masterstudiums an der Universität von Nantes unter der Leitung von Yves Henigfeld, Jean-François Nauleau und Alain Champagne durchgeführt wurde. Die diachrone Betrachtung hatte zum Ziel, das Handwerk der Ziegler oder Ziegelmacher vom 17. bis zum 20. Jh. im Kanton „Les Herbiers“ in der Vendée zu dokumentieren und zu analysieren (Chamarre, 2018 ; Chamarre, 2019). Die umfangreiche Auswertung kartographischer Unterlagen und des Quellenbestandes der Archive, die zunächst während des „Ancient Regime“ fortlaufend die lokalen kirchlichen und adeligen Dokumente aufnahmen und die später im 19. Jh. durch die Tätigkeiten der öffentlichen Verwaltung ergänzt wurden, hat es gestattet, die immer weiter zunehmende handwerkliche Tradition der Ziegeleiwirtschaft bis zur Mitte des 19. Jh. ausführlich zu dokumentieren. Anschließend kam es dann zu einem konstanten Rückgang der Ziegeleiwirtschaft bis zur Mitte des 20. Jh. Diese Dynamik, die sich bis in die Moderne fortsetzte, konnte nicht nur anhand der Erfassung und der Nachverfolgung zahlreicher kleiner, in einen lokalen ökonomischen Kontext eingebetteter Familienbetriebe, nachverfolgt werden, sondern sie wurde auch durch zunehmende ökonomische Zwänge bestimmt. Diese betrafen die Auswahl und Bevorzugung einer spezialisierten Produktion sowie politische und gesellschaftliche Veränderungen und Einschränkungen hinsichtlich der Beschäftigung von Frauen und Kindern. Die vorliegende Studie hat außerdem das Interesse an einer methodologischen Herangehensweise hervorgehoben, unter anderem hinsichtlich einer Beeinflussung durch die sich ändernde Materialwahl bei den zeitgenössischen Bauten sowie durch die Einbeziehung von Aussagen der ehemaligen Ziegeleibetreiber. Die zusammenfassende Darstellung der wichtigsten Ergebnisse dieser Studie gestattet es ferner, die am besten geeignete methodische Vorgehensweise bei der Untersuchung von neuzeitlichen und modernen Ziegeleien im Detail nachzuverfolgen.

Schlagwortindex: Vendée, Ziegelei, Moderne, Atelier, Ziegler, Archive, Methoden.

\section{La producción artesanal de tejas y ladrillos en el cantón de Les Herbiers (Vendée) en época contemporánea: metodología y perspectivas de investigación}

\begin{abstract}
Este artículo presenta los principales resultados de un estudio realizado durante dos años de máster en la Universidad de Nantes, bajo la supervisión de Yves Henigfeld, Jean-François Nauleau y Alain Champagne. Un enfoque diacrónico ha permitido comprender la producción artesanal de tejas y ladrillos, desde el siglo XVII hasta el siglo XX, en el cantón de Les Herbiers (Vendée) (Chamarre, 2018; Chamarre, 2019). Un examen exhaustivo de la cartografia y de los archivos, procedentes sucesivamente de los fondos señoriales y eclesiásticos del Antiguo Régimen y después de la administración pública del siglo XIX, permitió observar una creciente tradición artesanal hasta mediados del siglo XIX, antes de declive constante hasta mediados del siglo XX. Esta dinámica que continúa en la actualidad se percibe no sólo a través de la identificación y estudio de una multitud de pequeños talleres familiares, establecidos en un ámbito económico local, sino también a través de una creciente presión económica que influyó en la orientación hacia producciones especializadas o incluso en estrategias de empleo de mujeres y niños. El estudio también evalúa el interés metodológico de los enfoques a través de materiales de construcción contemporáneos y del testimonio de antiguos artesanos locales. Los principales resultados proporcionan una visión general de las pautas metodológicas más adecuadas para el estudio de la producción de contemporánea de tejas y ladrillos.
\end{abstract}

Palabras clave: Vendée, producción de tejas, época contemporánea, taller, explotación, artesanos tejeros, inventario, archivos, metodología. 